“(C) 2018 IEEE. Personal use of this material is permitted. Permission from IEEE must be obtained for all other uses, in any current or future media, including reprinting/republishing this material for advertising or promotional purposes, creating new collective works, for resale or redistribution to servers or lists, or reuse of any copyrighted component of this work in other works." 


\title{
Fractional Reverse Polarity Optical OFDM for High Speed Dimmable Visible Light Communications
}

\author{
Thomas Q. Wang and Xiaojing Huang, Senior Member, IEEE
}

\begin{abstract}
In this paper, fractional reverse polarity optical orthogonal frequency division multiplexing (FRPO-OFDM) is studied to enable dimming compatible visible light communications. The scheme combines a layered asymmetrically clipped optical orthogonal frequency division multiplexing (ACO-OFDM) sequence with an information-carrying brightness control sequence (BCS) in the form of $M$-ary pulse position modulation. We derive the expressions of the FRPO-OFDM signal and its achievable brightness level, and develop an effective detector which can recover information from both sequences based on maximum likelihood detection. We show that when the detector is to be implemented, the use of multi-layer ACO-OFDM imposes strong periodicity on the BCS, which leads to a trade-off between spectral efficiency and brightness resolution for dimming control. It is shown that high spectral efficiency can be achieved with practical dimming requirements. Simulation results show that the extra information carried by the BCS can be decoded with extremely low bit error rate and thus has negligible impacts on the demodulation of the ACO-OFDM signal, when the system non-linearity is not dominating.
\end{abstract}

Index Terms - Visible Light Communications, Digital Dimming Control, Optical Orthogonal Frequency Division Multiplexing, Intensity Modulation Direct Detection.

\section{INTRODUCTION}

W ITH the increasing use of the energy efficient white lighting light emitting diodes (LEDs), visible light communication (VLC) is emerging as a promising dual-use technology for indoor illumination and high speed communications. Unlike conventional radio frequency (RF) communications, VLC employs intensity modulation direct detection (IM/DD) to modulate the information to be transmitted onto the intensity of the light signals which are simultaneously used for illumination. This leads to the challenge that the transmitted intensity signals must meet the illumination constraint whilst being able to send the information to its destination [1].

Dimming control which delivers a comfortable and energy efficient lighting experience is an essential functionality of modern lighting systems. It can be implemented in a number of approaches. The most straightforward is known as the analog dimming which adjusts the brightness level through directly controlling the strength of the forward current. This is possibly the simplest solution which ideally leads to continuous adjustment, but may lead to noticeable chromaticity

This work was presented in part at the IEEE 85th Vehicular Technology Conference Spring, (VTC 2017 Spring). This work was supported under an Australian Research Council's (ARC) Discovery funding scheme (DP 160101693)

The authors are with Global Big Data Technologies Centre, University of Technology Sydney, Sydney, Australia e-mail: \{qian.wang, xiaojing.huang\}@uts.edu.au shift [2]. Alternatively, digital dimming, also known as pulse width modulation [2], can be applied, in which the forward current is in the form of rectangular pulses and the brightness level is controlled through varying their duty cycle [3]. Typical digital dimming schemes used in VLC include variable on-off keying (VOOK), variable pulse position modulation (VPPM) and multiple pulse position modulation (MPPM) [3]. As these schemes are easy to implement and result in reduced chromaticity shift, they are preferred in industry standards [4].

Optical orthogonal frequency division multiplexing (OFDM) is increasingly being used in VLC because of its robustness to inter-symbol interference and high energy and spectral efficiencies [5]. However, original optical OFDM schemes, such as asymmetrically clipped optical OFDM (ACO-OFDM) [6], DC-biased optical OFDM (DCO-OFDM) [7], asymmetrically clipped DC biased optical OFDM (ADO-OFDM) [8] and Unipolar OFDM (U-OFDM) [9], are initially proposed to create real non-negative signals that can be used in IM/DD systems. Therefore, improvements must be made to the original schemes if the compatibility to dimming control is to be achieved. Recent research has shown a series of dimming compatible OFDM schemes using either analog [10][11][12] or digital approaches [13][14]. The analog approach, known as signal shaping [10], produces a wide range of brightness levels through directly adjusting the biasing and scaling of the optical OFDM signals. This approach was first studied for ACO- and DCO-OFDM [10] and later extended to asymmetrical hybrid optical OFDM (AHO-OFDM) [11] and enhanced DCO-OFDM [12]. It is shown that given a proper direct current (DC) bias and scaling coefficient, the communication performance of optical OFDM (e.g. the bit error rate (BER)) can be upheld for different dimming levels [10]. The challenge of implementing signal shaping stems from the absence of an efficient analytical approach to evaluating the bias and scaling coefficient [10]. Alternatively, dimmable OFDM schemes are recently proposed using digital dimming control [13][14]. In both of the methods [13][14], a periodic binary brightness control sequence (BCS) is employed to control the brightness with its duty cycle. In [13], this sequence is directly multiplied with an optical OFDM signal to form a sampled version of the signal. However, in order to recover the OFDM signal, the sequence must have very high frequency which, in fact, cannot be achieved by off-the-shelf LED drivers. In [14], reverse polarity optical OFDM (RPO-OFDM) is proposed in which the two levels of the BCS, corresponding to the off and on states, are used as both the pedestals of the unipolar OFDM signals and the bounds of the dynamic range of the 
resulting signals. The durations of the on and off states are respectively set to be integer multiples of that of the unipolar OFDM symbol, depending on the desired brightness. Because of its periodicity, the BCS used in RPO-OFDM carries no information.

The fast Fourier transform (FFT) pair employed in the optical OFDM transceivers leads to large number of samples at the receiver from which a hidden informative waveform may be detected with high reliability [15]. This stimulates the use of an information-carrying BCS to form a novel dimming compatible optical OFDM scheme, fractional RPO-OFDM (FRPO-OFDM) [16]. Unlike the existing digital dimming schemes [13][14], FRPO-OFDM transmits information using two simultaneous data streams: a high data rate optical OFDM sequence and a low rate BCS with high reliability [16]. In the receiver, a two-stage demodulation is performed, where the BCS is demodulated in the first stage and the optical OFDM, in the second stage based on the decoded BCS. The use of an informative BCS is of significant practical value to VLC systems. This is because certain types of data, such as control signals, are demanding on reliability other than speed, which perfectly fit the characteristic of BCS. As a result, the payload data alone can be loaded onto the high speed optical OFDM sequence. This is in contrast with the existing schemes, where the control signals must be transmitted with low rate in the optical OFDM component, causing loss of efficiency.

In this paper, we present an in-depth investigation on FRPOOFDM. A series of improvements have been made to the FRPO-OFDM introduced in [16] to enhance the spectral efficiency. These include a new BCS using Gray coded $M$ ary pulse position modulation $(M-\mathrm{PPM})$ for brightness control and the use of layered ACO-OFDM [17]. The new BCS enables multiple extra bits to be transmitted in an OFDM symbol whilst avoiding flickering caused by long strings of zeros or ones. We use ACO- other than DCO-OFDM because the former has much lower expected value. This means that when combined with the BCS, ACO-OFDM leads to a much wider range of illumination levels than DCO-OFDM does [14]. The loss of the spectral efficiency of the ACO-OFDM can be compensated using multi-layer structure. It has been shown that the spectral efficiency of the layered ACO-OFDM improves rapidly with increasing number of layers ${ }^{1}$ [17]. Besides these improvements, the new contributions of this paper also include

- The derivation of the measured and perceived brightness level achieved by FRPO-OFDM in which an approach to evaluating the probability density function (PDF) of the layered ACO-OFDM is developed.

- The development of an effective detector to retrieve the information from both the sequences.

- The study of a trade-off between the spectral efficiency and brightness resolution. We show that in order to decode FRPO-OFDM signals, strong periodicity has to be imposed on the BCS, which reduces the number of brightness steps with increasing layers.

\footnotetext{
${ }^{1}$ For the same reason, other optical OFDM schemes based on asymmetrical clipping, such as hybrid ACO-OFDM [18] and enhanced U-OFDM [19] can also be used in FRPO-OFDM if given corresponding detectors.
}

This paper is organized as follows. The signal design and dimming control are described in II. The detector is developed in Section III. We present the simulation results in Section IV and conclude the paper in Section V.

\section{Signal Design And Dimming Control}

\section{A. Fractional RPO-OFDM}

FRPO-OFDM distinguishes from the original RPO-OFDM [14] in two major aspects. First, the periodic BCS is replaced by an information-carrying one in the form of $M_{\mathrm{PPM}}$-PPM, where $M_{\text {PPM }}$ denotes the order of the PPM. Thus, the BCS used in FRPO-OFDM will have $M_{\mathrm{PPM}}$ possible waveforms, each carrying $\log _{2} M_{\mathrm{PPM}}$ information bits. Second, the BCS is configured to have a size identical to that of an ACO-OFDM symbol. Therefore, unlike RPO-OFDM, the transition between the on and off states in a BCS will be located within each OFDM symbol and the dimming/brightness level depends on the fractions the two states take up. As shown in Fig. 1, an FRPO-OFDM symbol is constructed using three modules: 1) a layered ACO-OFDM modulator, 2) an $M_{\mathrm{PPM}}$-PPM modulator and 3) a combining module.

1) Layered ACO-OFDM: An L-layer ACO-OFDM modulator, $L \leq \log _{2} N$, which converts complex bipolar symbols, $\boldsymbol{X}=\left[0, X_{1}, \cdots, X_{N / 2-1}, 0, X_{N / 2-1}^{*}, \cdots, X_{1}^{*}\right]^{T}$ to real nonnegative intensity signals, $s^{(L)}=\left[s_{0}^{(L)}, \cdots, s_{N-1}^{(L)}\right]^{T}$, consists of $L$ single layer ACO-OFDM modulators, where independent $M_{\mathrm{OFDM}}$-QAM symbols, $X_{1}, \cdots, X_{N / 2-1}$, with $E\left(\left|X_{n}\right|^{2}\right)=\epsilon$, are loaded with Hermitian symmetry. Here, $N$ denotes the size of the IFFT at each modulator and the superscripts, $[\cdot]^{T}$ and $[\cdot]^{*}$, represent the vector/matrix transpose and complex conjugate, respectively. Unlike conventional ACO-OFDM [6], the inputs to the $l$ th, $l \leq L$, modulator, denoted by $X_{l}=$ $\left[X_{l, 0}, X_{l, 1}, \cdots, X_{l, N-1}\right]^{T}$, is related with the input symbol, $\boldsymbol{X}$, by

$$
X_{l, n}=\left\{\begin{array}{l}
X_{n}, \quad \text { if } n \in G_{l} \\
0, \quad \text { otherwise }
\end{array},\right.
$$

where $\mathcal{G}_{l}$ denotes the $l$ th subcarrier group (SCG) which includes those subcarriers with indexes dividable by $2^{l-1}$ but not dividable by $2^{l}$ [20]. Eq. (1) confirms that the conventional ACO-OFDM corresponds to the first layer modulator which loads symbols onto the odd subcarriers.

The IFFT of the $l$ th modulator generates a sequence, $x_{l}=$ $\left[x_{l, 0}, x_{l, 1}, \cdots, x_{l, N-1}\right]^{T}$, given by

$$
x_{l, k}=\frac{1}{\sqrt{N}} \sum_{n=0}^{N-1} X_{l, n} e^{j \frac{2 \pi}{N} k n}, \text { for } 0 \leq k \leq N-1
$$

which produces the output of the modulator, $s_{l}=$ $\left[s_{l, 0}, s_{l, 1}, \cdots, s_{l, N-1}\right]^{T}$, through clipping its negative value at zero to give

$$
s_{l, k}=\left(x_{l, k}+\left|x_{l, k}\right|\right) / 2=\left\{\begin{array}{ll}
x_{l, k}, & \text { if } x_{l, k} \geq 0 \\
0, & \text { otherwise }
\end{array} .\right.
$$




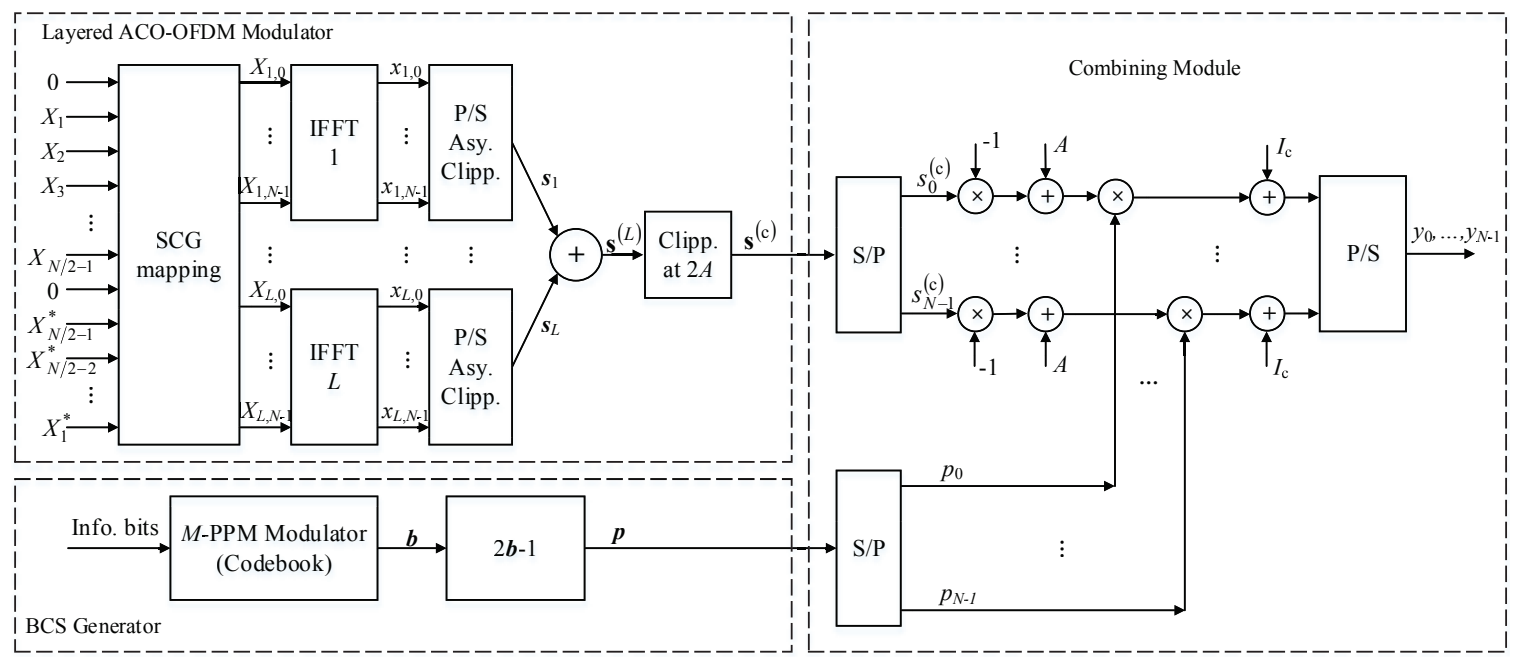

Fig. 1. Block diagram of FRPO-OFDM.

The output of the $L$-layer ACO-OFDM modulator, $s^{(L)}=$ $\left[s_{0}^{(L)}, \cdots, s_{N-1}^{(L)}\right]^{T}$, is the sum of those of all single layer modulators and thus can be expressed as

$$
s_{k}^{(L)}=\sum_{l=1}^{L} s_{l, k}, \text { for } 0 \leq k \leq N-1,
$$

with the electrical power, $P_{\text {elec }}$, given $b^{2}{ }^{2}$ [21]

$$
P_{\text {elec }}=E\left(\sum_{l=1}^{L} s_{l, k}\right)^{2}=\frac{1}{2} \sum_{l=1}^{L} \sigma_{l}^{2}+\frac{1}{\pi} \sum_{l_{1} \neq l_{2}}^{L} \sigma_{l_{1}} \sigma_{l_{2}},
$$

where $\sigma_{l}^{2}=E\left(x_{l, k}^{2}\right)$, denotes the electrical power of $x_{l, k}$ and equals $\epsilon / 2^{l}$. Therefore, the average electrical energy per bit, $E_{\mathrm{b} \text {,elec }}$, is given by $E_{\mathrm{b} \text {,elec }}=P_{\text {elec }} / r_{\mathrm{b}}$, where $r_{\mathrm{b}}$ denotes the bit rate of the layered ACO-OFDM signal [8].

2) Generation of BCS and Digital Dimming: The BCS, $\boldsymbol{b}=\left[b_{0}, b_{1}, \cdots, b_{N-1}\right]^{T}$, is a binary sequence, where $b_{k}=0$ and 1 correspond to the off and on states, respectively. In order to carry $\log _{2} M_{\mathrm{PPM}}$ bits using PPM, for a given brightness level, $M_{\text {PPM }}$ codewords must be included in the corresponding codebook of BCS, each consisting of $N$ bits and assigning the on/off state to different portions of the BCS. In line with conventional PPM, we divide the BCS into $M_{\mathrm{PPM}}$ groups. Then, the BCS can be rewritten as $\boldsymbol{b}=\left[\boldsymbol{G}_{0}^{T}, \boldsymbol{G}_{2}^{T}, \cdots, \boldsymbol{G}_{M_{\mathrm{PPM}}-1}^{T}\right]^{T}$ where each group, denoted by $\boldsymbol{G}_{j}, j=0, \cdots, M_{\mathrm{PPM}}-1$, comprises $N / M_{\text {PPM }}$ bits taking identical value. Therefore, the information carried by the BCS can be conveyed through the on/off state of each group.

\footnotetext{
${ }^{2}$ The derivation of (5) is based on the assumption that the outputs of the IFFTs are Gaussian distributed. The distribution of $s_{l, k}, 1 \leq l \leq L$, depends on the number of subcarriers included in the $l$ th SCG. When it is greater than 16 , the output of the IFFT, $x_{l, k}$, can be well approximated as a zero-mean Gaussian distributed random variable [21]. For the case where the number is less than 16, the central limit theorem does not apply. However, as very few subcarriers are loaded, the power of $x_{l, k}$ will be extremely small compared to those resulted from other SCGs, Thus, the error caused by a Gaussian approximation will be negligible in the PDF of $s_{k}^{(L)}$.
}

The brightness level generated by the BCS depends on the number of groups, $c$, configured at on state in each codeword. In order to produce constant brightness regardless of the information to be transmitted, for a given brightness level, we fix this number in all the codewords in the corresponding codebook. As $M_{\mathrm{PPM}}$ groups are included in a BCS, $M_{\mathrm{PPM}}+1$ brightness steps are potentially supported, among which $c=1, \cdots, M_{\mathrm{PPM}}-1$ leads to at least $M_{\mathrm{PPM}}$ possible waveforms to be chosen from as the codewords in each resulting brightness level. Therefore, information can be transmitted in these steps. The other two steps, (i.e. $c=0$ and $M_{\mathrm{PPM}}$ ), however, correspond to the all zero and all one BCSs that cannot be used for communications.

For the $c$ th brightness step, $1 \leq c \leq M_{\mathrm{PPM}}-1$, there are $\left(\begin{array}{c}M_{\mathrm{PPM}} \\ c\end{array}\right)$ possible waveforms from which $M_{\mathrm{PPM}}$ codewords are selected to form the codebook corresponding to this brightness level. Here we introduce an illustrative codebook, $C^{\left(N, M_{\mathrm{PPM}}, c\right)}=\left\{C_{0}^{\left(N, M_{\mathrm{PPM}}, c\right)}, C_{1}^{\left(N, M_{\mathrm{PPM}}, c\right)}, \cdots, C_{M_{\mathrm{PPM}}-1}^{\left(N, M_{\mathrm{PPM}}, c\right)}\right\}$, in which the $i$ th codeword is at on state only in its $(i)_{M_{\mathrm{PPM}}}$ th, $\cdot$ $\cdot,(i+c-1)_{M_{\mathrm{PPM}}}$ th groups, where $(\cdot)_{M_{\mathrm{PPM}}}$ denotes modulo $M_{\mathrm{PPM}}$. Such a codebook with $c=3$ is shown in Table I, where $N=16$ samples are grouped into $M_{\mathrm{PPM}}=8$ groups. Then each group consists of $N / M_{\mathrm{PPM}}=2$ bits. It can be seen that the codebook consists of $M_{\mathrm{PPM}}=8$ codewords which are the circular shifts of each other. We can also see that each codeword has two neighbouring codewords which distinguish from them with four bits (two groups) $\left(C_{0}^{(16,8,3)}\right.$ is the neighbour of $C_{1}^{(16,8,3)}$ and $C_{7}^{(16,8,3)}$ ). The maximum distinction is seen between any two codewords $M_{\mathrm{PPM}} / 2$ apart from each other for $c=3$. These enable the use of Gray codes to map the information to be transmitted to the codeword. In the last column of the table, the information bits corresponding to each codeword are listed showing the one-bit difference between neighbouring codewords.

3) Combining Module: The combining module performs element-wise multiplications and additions to the layered ACO-OFDM samples and BCS, yielding the FRPO-OFDM 
TABLE I

AN ILLUSTRATIVE CODEBOOK WITH $N=16, M_{\mathrm{PPM}}=8$ AND $c=3$

\begin{tabular}{|c|c|c|c|c|c|c|c|c|c|}
\hline & $\boldsymbol{G}_{0}^{T}$ & $\boldsymbol{G}_{1}^{T}$ & $\boldsymbol{G}_{2}^{T}$ & $G_{3}^{T}$ & $G_{4}^{T}$ & $G_{5}^{T}$ & $\boldsymbol{G}_{6}^{T}$ & $\boldsymbol{G}_{7}^{T}$ & Info \\
\hline$C_{0}^{(16,8,3)}$ & 11 & 11 & 11 & 00 & 00 & 00 & 00 & 00 & 000 \\
\hline$C_{1}^{(16,8,3)}$ & 00 & 11 & 11 & 11 & 00 & 00 & 00 & 00 & 001 \\
\hline$C_{2}^{(16,8,3)}$ & 00 & 00 & 11 & 11 & 11 & 00 & 00 & 00 & 011 \\
\hline$C_{3}^{(16,8,3)}$ & 00 & 00 & 00 & 11 & 11 & 11 & 00 & 00 & 010 \\
\hline$C_{4}^{(16,8,3)}$ & 00 & 00 & 00 & 00 & 11 & 11 & 11 & 00 & 110 \\
\hline$C_{5}^{(16,8,3)}$ & 00 & 00 & 00 & 00 & 00 & 11 & 11 & 11 & 111 \\
\hline$C_{6}^{(16,8,3)}$ & 11 & 00 & 00 & 00 & 00 & 00 & 11 & 11 & 101 \\
\hline$C_{7}^{(16,8,3)}$ & 11 & 11 & 00 & 00 & 00 & 00 & 00 & 11 & 100 \\
\hline
\end{tabular}

samples,

$$
y_{k}=p_{k}\left(-s_{k}^{(\mathrm{c})}+A\right)+I_{\mathrm{c}}, \text { for } 0 \leq k \leq N-1
$$

where $I_{\mathrm{c}}, I_{\mathrm{c}}>A$, and $2 A, A>0$, denote the center value and the width of the signal's dynamic range, respectively. $s_{k}^{(\mathrm{c})}$ is obtained by clipping $s_{k}^{(L)}$ at $2 A$, i.e.

$$
s_{k}^{(\mathrm{c})}=\left\{\begin{array}{ll}
s_{k}^{(L)}, & s_{k}^{(L)}<2 A \\
2 A, & s_{k}^{(L)} \geq 2 A
\end{array},\right.
$$

and sequence $\boldsymbol{p}=\left[p_{0}, p_{1}, \cdots, p_{N-1}\right]^{T}$ is related to the BCS by $p_{k}=2 b_{k}-1$.

As shown in (6), the term, $-s_{k}^{(\mathrm{c})}+A$, is a non-positive going sequence biased at $A$, representing the on state. Thus, the maximum and minimum values of this sequence will be $A$ and $-A$, respectively, i.e., this sequence is constrained between $\pm A$. When multiplied with $p_{k}$ which equals either 1 or -1 , the sequence will either remain at the on state or be flipped to the off state, resulting in the term, $p_{k}\left(-s_{k}^{(\mathrm{c})}+A\right)$, a sequence centered at 0 with a dynamic range of $2 A$. A DC bias, $I_{\mathrm{c}}$, is then superimposed, leading to the FRPO-OFDM signal ranging from $I_{\mathrm{L}}=I_{\mathrm{c}}-A$ to $I_{\mathrm{H}}=I_{\mathrm{c}}+A$, where $I_{\mathrm{L}}$ and $I_{\mathrm{H}}$ denote the minimum and maximum values of the FRPOOFDM signal, respectively. The resulting signal is emitted by the LEDs after being added a cyclic prefix and digitalto-analogue conversion (DAC).

Fig. 2 demonstrates two successive FRPO-OFDM symbols, where Fig. 2(a) plots the original single layer ACO-OFDM signal, and Fig. 2(b) and (c) the FRPO-OFDM signal using the illustrative codebook, $C^{(16,8, c)}$. For Symbol 1, information bits 000 is transmitted by the BCS using codeword $C_{0}^{(16,8, c)}$ and for Symbol 2, 110 using $C_{4}^{(16,8, c)}$. The third and sixth brightness steps corresponding to $c=3$ and 6 are plotted in Fig. 2(b) and (c), respectively, where the on and off states are illustrated. Comparing Fig. 2(a) with (b) and (c), we can see that because of the use of a BCS, the average optical power of the FRPOOFDM is different from that of the original ACO-OFDM. Also, the ACO-OFDM signal is seen completely contained
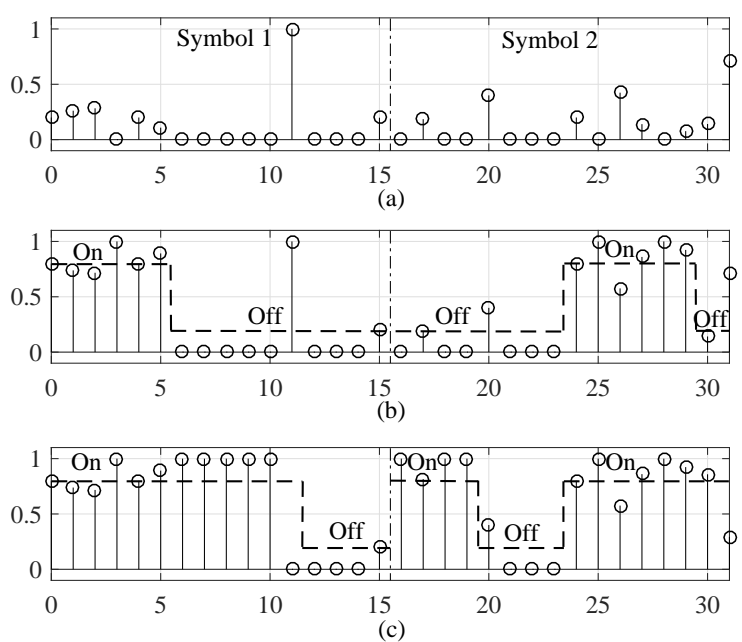

Fig. 2. A demonstration of the FRPO-OFDM signal, (a) Single layer ACOOFDM, (b) FRPO-OFDM with $c=3$, and (c) FRPO-OFDM with $c=6$.

in the dynamic range regardless of the brightness level ${ }^{3}$. This addresses the issue raised in analog dimming. From Fig. 2(b) and (c), it can be seen that the sixth level generates higher optical power than the third level. This is consistent with the design of the BCS.

\section{B. Measured and Perceived Brightness}

Now we derive the expression of the measured and perceived brightness. We assume the dynamic range of the FRPOOFDM signal is included in that of the LED luminaire. Therefore, the electrical-to-optical conversion is linear. In line with [14], the measured brightness level (MBL) is given by

$$
\mathrm{BL}_{\mathrm{m}}=\frac{I_{\mathrm{avg}}-I_{\mathrm{L}}}{I_{\mathrm{H}}-I_{\mathrm{L}}}
$$

where $I_{\text {avg }}$ denotes the average current input to the LED. For the $c$ th brightness step, there are $c$ groups set at the on state and $M_{\mathrm{PPM}}-c$ groups, the off state. As the layered ACO-OFDM signal is non-positive going at the on state and non-negative going at the off-state, the average current can be expressed as

$$
I_{\mathrm{avg}}=\frac{c}{M_{\mathrm{PPM}}}\left(I_{\mathrm{H}}-\bar{s}_{k}^{(\mathrm{c})}\right)+\frac{M_{\mathrm{PPM}}-c}{M_{\mathrm{PPM}}}\left(I_{\mathrm{L}}+\bar{s}_{k}^{(\mathrm{c})}\right),
$$

where $\bar{s}_{k}^{(\mathrm{c})}$ denotes the expected value of the clipped $L$-layer ACO-OFDM signal, $s_{k}^{(\mathrm{c})}$.

The expected value, $\bar{s}_{k}^{(\mathrm{c})}$, depends on the PDF of $s_{k}^{(L)}$ which is the sum of $L$ single-layer ACO-OFDM signals, each having a PDF given by [21]

$$
f_{s_{l, k}}(s)=\frac{1}{2} \delta(s)+\frac{1}{\sqrt{2 \pi} \sigma_{l}} \exp \left(-\frac{s^{2}}{2 \sigma_{l}^{2}}\right) \mathrm{I}(s), \quad 1 \leq l \leq L,
$$

where $\delta(s)$ denotes the Dirac delta function and $\mathrm{I}(\cdot)$, the indicator function returning one with positive inputs and zero

\footnotetext{
${ }^{3}$ Note that an LED must be modulated by analogue electrical signals that fall into its dynamic range, and that the analogue signals typically have a wider dynamic range than that of their samples in digital domain [23]. Therefore, a margin of the dynamic range must be applied in the digital domain.
} 
otherwise. As a result, we implement a recursive approach to deriving the PDF of $s_{k}^{(L)}$, where the relationship between the PDFs, $f_{s_{k}^{(L)}}(s)$ and $f_{s_{k}^{(L-1)}}(s)$ are established as

$$
\begin{aligned}
f_{s_{k}^{(L)}}(s)= & \frac{1}{2^{L}} \delta(s)+\frac{1}{2^{L}} f_{s_{L, k}}\left(s \mid s_{L, k}>0\right) \\
& +\left(\frac{1}{2}-\frac{1}{2^{L}}\right) f_{s_{k}^{(L-1)}}\left(s \mid s_{k}^{(L-1)}>0\right) \\
& +\left(\frac{1}{2}-\frac{1}{2^{L}}\right) f_{s_{k}^{(L-1)}+s_{L, k}}\left(s \mid s_{k}^{(L-1)}>0, s_{L, k}>0\right),
\end{aligned}
$$

where $\quad f_{s_{L, k}}\left(s \mid s_{L, k}>0\right), \quad f_{s_{k}^{(L-1)}}\left(s \mid s_{k}^{(L-1)}>0\right) \quad$ and $f_{s_{k}^{(L-1)}+s_{L, k}}\left(s \mid s_{k}^{(L-1)}>0, s_{L, k}>0\right)^{k}$ denote the conditional PDFs of $s_{L, k}, s_{k}^{(L-1)}$, and $s_{k}^{(L-1)}+s_{L, k}$, given by

$$
\begin{gathered}
f_{s_{L, k}}\left(s \mid s_{L, k}>0\right)=\frac{2}{\sqrt{2 \pi} \sigma_{L}} \exp \left(-\frac{s^{2}}{2 \sigma_{L}^{2}}\right), \quad s>0, \\
f_{s_{k}^{(L-1)}}\left(s \mid s_{k}^{(L-1)}>0\right)=\frac{1}{1-2^{-(L-1)}} f_{s_{k}^{(L-1)}}(s), \quad s>0 .
\end{gathered}
$$

and

$$
\begin{aligned}
& f_{s_{k}^{(L-1)}+s_{L, k}}\left(s \mid s_{k}^{(L-1)}>0, s_{L, k}>0\right) \\
& =\left(\frac{1}{1-2^{-(L-1)}}\right) \int_{0}^{s} f_{s_{k}^{(L-1)}}(s-z) f_{s_{L, k}}\left(z \mid s_{L, k}>0\right) \mathrm{d} z, s>0,
\end{aligned}
$$

respectively. See Appendix A for the derivation of (11)-(14). Eqs. (11)-(14) indicate that the PDF of the L-layer signal depends on that of the $(L-1)$-layer signal, $s_{k}^{(L-1)}=\sum_{l=1}^{L-1} s_{l, k}$, and the signal of the $L$ th layer, $s_{L, k}$. Therefore, it can be derived numerically using the following procedure:

1) Initialization: The PDFs of $s_{i, k}, i=1,2$ and $f_{s_{k}^{(1)}}(s)$, are given by

$$
\begin{aligned}
f_{s_{i, k}}(s) & =\frac{1}{2} \delta(s)+\frac{1}{\sqrt{2 \pi} \sigma_{i}} \exp \left(-\frac{s^{2}}{2 \sigma_{i}^{2}}\right) \mathrm{I}(s) . \\
f_{s_{k}(1)}(s) & =f_{s_{1, k}}(s) .
\end{aligned}
$$

2) Calculation: While $l \leq L-1$, evaluate $f_{s_{k}^{(l+1)}}(s)$ using $f_{s_{k}^{(l)}}(s)$ and $f_{s_{l+1, k}}\left(s \mid s_{l+1, k}>0\right)$.

The proposed procedure cannot return the exact PDFs in closed-form expressions when more than two layers are employed in ACO-OFDM. This is because given the PDFs in the initialization of the procedure, the PDF of double-layer ACO-OFDM signal can be derived in closed-form as

$$
\begin{aligned}
f_{s_{k}^{(2)}}(s)= & \frac{1}{4} \delta(s)+\frac{1}{2 \sqrt{\pi \epsilon}} \exp \left(-\frac{s^{2}}{\epsilon}\right)+\frac{1}{\sqrt{2 \pi \epsilon}} \exp \left(-\frac{2 s^{2}}{\epsilon}\right) \\
& +\sqrt{\frac{2}{3 \pi \epsilon}} \exp \left(-\frac{2 s^{2}}{3 \epsilon}\right)\left[Q\left(-\sqrt{\frac{2}{3 \epsilon}} s\right)-Q\left(2 \sqrt{\frac{2}{3 \epsilon}} s\right)\right],
\end{aligned}
$$

where $Q(\cdot)$ denotes the Gaussian $\mathrm{Q}$ function. This means that the calculation for three-layer signal involves the integral

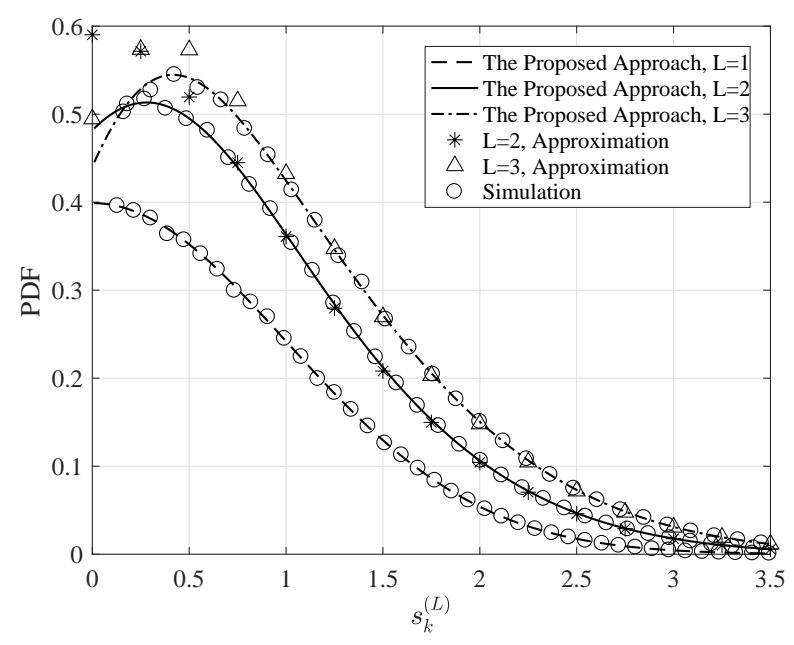

Fig. 3. PDF of the layered ACO-OFDM signal.

of $\mathrm{Q}$ function and exponential functions which may become intractable, leading to the PDF of $l$-layer ACO-OFDM, $l \geq 3$, difficult to be expressed in closed form.

The Gaussian Q function can be approximated by exponential functions as [25]

$$
Q(t) \approx \frac{1}{12} \exp \left(-\frac{1}{2} t^{2}\right)+\frac{1}{4} \exp \left(-\frac{2}{3} t^{2}\right),
$$

where the approximation is tight when $t \geq 0.5$. Therefore, inserting (17) into (16) yields an approximation of $f_{s_{k}^{(2)}}(s)$ as

$$
\begin{aligned}
f_{s_{k}^{(2)}}(s) & \approx \frac{1}{4} \delta(s)+\left(\frac{1}{2 \sqrt{\pi \epsilon}}-\frac{1}{12} \sqrt{\frac{2}{3 \pi \epsilon}}\right) \exp \left(-\frac{s^{2}}{\epsilon}\right) \\
& +\left(\frac{1}{\sqrt{2 \pi \epsilon}}-\frac{1}{12} \sqrt{\frac{2}{3 \pi \epsilon}}\right) \exp \left(-\frac{2 s^{2}}{\epsilon}\right) \\
& +\sqrt{\frac{2}{3 \pi \epsilon}} \exp \left(-\frac{2 s^{2}}{3 \epsilon}\right)-\frac{1}{4} \sqrt{\frac{2}{3 \pi \epsilon}} \exp \left(-\frac{10 s^{2}}{9 \epsilon}\right) \\
& -\frac{1}{4} \sqrt{\frac{2}{3 \pi \epsilon}} \exp \left(-\frac{22 s^{2}}{9 \epsilon}\right) .
\end{aligned}
$$

As the approximate PDF, (18), consists of only exponential functions in its continuous part, the use of it in the calculation for three-layer ACO-OFDM will produce terms of only exponential and $\mathrm{Q}$ functions which can be then approximated using (17) to calculate for four-layer signals. Thus, the PDFs of up to $L$-layer ACO-OFDM can be rewritten in closed form.

Fig. 3 shows the continuous part of the PDF of the layered ACO-OFDM signal obtained using the proposed procedure and its approximation, where $N=256$ subcarriers are assumed to be loaded by the symbols with $\epsilon=2$. The PDFs of the single-layer, two-layer and three-layer signals are plotted against the simulation results. From this figure, we can see good match between the derived PDFs and the simulation, which verifies the procedure. We can also see from Fig. 3 that all the three PDFs have long tails gradually decaying with increasing value of the signals. For sufficiently large signal values, e.g. $s_{k}^{(L)}>0.5$, the PDF of three-layer signal 
is constantly the greatest, whereas that for single-layer, the smallest among the three signals. Recalling that given the width of the dynamic range, $2 A$, the area circulated by $s_{k}^{(L)}=2 A$, the horizontal axis and the tail of a signal's PDF represents the probability of the signal exceeding the dynamic range, it can be seen that the three-layer signal has the largest probability of being clipped, followed by the two-layer signal. This is in line with the intuition that a multi-layer signal is more likely to take large values. The long tails of the PDFs result in the clippings at the boundaries of the signal's dynamic range, (see (7)), and lead to the expected value, $\bar{s}_{k}^{(\mathrm{c})}$, given by

$$
\bar{s}_{k}^{(\mathrm{c})}=0 \times \frac{1}{2^{L}}+\int_{0_{+}}^{2 A} s f_{s_{k}^{(L)}}(s) \mathrm{d} s+2 A \int_{2 A}^{\infty} f_{s_{k}^{(L)}}(s) \mathrm{d} s .
$$

The perceived brightness level $(\mathrm{PBL}), \mathrm{BL}_{\mathrm{p}}$, is related to the MBL by $\mathrm{BL}_{\mathrm{p}}=\sqrt{\mathrm{BL}_{\mathrm{m}}}[14]$.

\section{Signal Detection}

In this section, we derive a simple and effective detector that recovers the information from both the layered ACO-OFDM signal and the BCS. Because of the DC offset existing between the transmitter and receiver [24], the received signal can be expressed as

$r_{k}=y_{k}+w_{k}=p_{k}\left(-s_{k}^{(\mathrm{c})}+A\right)+I_{\mathrm{c}}^{\prime}+w_{k}$, for $0 \leq k \leq N-1$,

where $I_{\mathrm{c}}^{\prime}$ and $w_{k}$ denote the DC-bias and noise at the receiver, respectively. In this paper, only shot noise is considered, which is induced by the ambient light. Thus the noise, $w_{k}, 0 \leq k \leq$ $N-1$, is assumed to be independently Gaussian distributed with zero mean and power of $\sigma_{\mathrm{w}}^{2}$.

\section{A. Maximum Likelihood Detection and Periodic BCSs}

The demodulation of FRPO-OFDM signals can be modelled as an $M_{\mathrm{PPM}}$-ary hypothesis test with nuisance parameters, $s^{(c)}=\left[s_{0}^{(c)}, s_{1}^{(c)}, \cdots, s_{N-1}^{(c)}\right]^{T}$ and $I_{\mathrm{c}}^{\prime}$. We denote the $M_{\mathrm{PPM}}$ hypotheses by $\mathcal{H}_{i}, i=1, \cdots, M_{\mathrm{PPM}}$, where conditioned on each hypothesis, a corresponding codeword is assumed to be carried by the BCS, $\boldsymbol{b}^{\mathcal{H}_{i}}=\left[b_{0}^{\mathcal{H}_{i}}, b_{1}^{\mathcal{H}_{i}}, \cdots, b_{N-1}^{\mathcal{H}_{i}}\right]^{T}$, in the form of the vector $\boldsymbol{p}^{\mathcal{H}_{i}}=\left[p_{0}^{\mathcal{H}_{i}}, p_{1}^{\mathcal{H}_{i}}, \cdots, p_{N-1}^{\mathcal{H}_{i}}\right]^{T}$. In addition, we denote the estimates of the nuisance parameters conditioned on $\mathcal{H}_{i}, i=1, \cdots, M_{\mathrm{PPM}}$, by $\hat{\boldsymbol{s}}_{\mathcal{H}_{i}}^{(\mathrm{c})}=\left[\hat{s}_{0, \mathcal{H}_{i}}^{(\mathrm{c})}, \cdots, \hat{s}_{N-1, \mathcal{H}_{i}}^{(\mathrm{c})}\right]^{T}$ and $\hat{I}_{\mathrm{c}}^{\mathcal{H}_{i}}$, respectively. Define $M_{\mathrm{PPM}}$ auxiliary vectors, $\boldsymbol{u}^{\mathcal{H}_{i}}$, given by $\boldsymbol{u}^{\mathcal{H}_{i}}=\boldsymbol{r}-A \boldsymbol{p}^{\mathcal{H}_{i}}$, where $\boldsymbol{r}=\left[r_{0}, \cdots, r_{N-1}\right]^{T}$. Then, conditioned on $\mathcal{H}_{i}$ and the corresponding estiamtes, $\hat{I}_{\mathrm{c}}^{\mathcal{H}_{i}}$ and $\hat{\boldsymbol{s}}_{\mathcal{H}_{i}}^{(\mathrm{c})}$, the joint PDF of $\boldsymbol{u}^{\mathcal{H}_{i}}$, also known as the likelihood function conditioned on $\mathcal{H}_{i}$, can be expressed as

$$
\begin{aligned}
& f_{\boldsymbol{u}^{\mathcal{H}_{i}}}\left(u_{0}^{\mathcal{H}_{i}}, \cdots, u_{N-1}^{\mathcal{H}_{i}} \mid \hat{I}_{\mathrm{c}}^{\mathcal{H}_{i}}, \hat{\boldsymbol{s}}_{\mathcal{H}_{i}}^{(\mathrm{c})}, \mathcal{H}_{i}\right) \\
= & \left(\frac{1}{\sqrt{2 \pi} \sigma_{\mathrm{w}}}\right)^{N} \exp \left[-\frac{1}{2 \sigma_{\mathrm{w}}^{2}} \sum_{k=0}^{N-1}\left(u_{k}^{\mathcal{H}_{i}}+p_{k}^{\mathcal{H}_{i}} \hat{s}_{k, \mathcal{H}_{i}}^{(\mathrm{c})}-\hat{I}_{\mathrm{c}}^{\mathcal{H}_{i}}\right)^{2}\right] .
\end{aligned}
$$

Given the received signal, $\boldsymbol{r}$, the estimates under various hypotheses typically leads to different values for the likelihood functions. The maximum likelihood (ML) detector returns the hypothesis that maximizes the likelihood function, (21), as the decoded BCS, i.e.

$$
\begin{aligned}
\hat{\boldsymbol{b}} & =\arg \max _{i}\left[f_{\boldsymbol{u}^{\mathcal{H}_{i}}}\left(u_{0}^{\mathcal{H}_{i}}, \cdots, u_{N-1}^{\mathcal{H}_{i}} \mid \hat{I}_{\mathrm{c}}^{\mathcal{H}_{i}}, \hat{\boldsymbol{s}}_{\mathcal{H}_{i}}^{(\mathrm{c})}, \mathcal{H}_{i}\right)\right] \\
& =\arg \min _{i} \sum_{k=0}^{N-1}\left(u_{k}^{\mathcal{H}_{i}}+p_{i}^{\mathcal{H}_{i}} \hat{\boldsymbol{s}}_{k, \mathcal{H}_{i}}^{(\mathrm{c})}-\hat{I}_{\mathrm{c}}^{\mathcal{H}_{i}}\right)^{2} .
\end{aligned}
$$

Now we derive the estimates of the ACO-OFDM samples and DC bias under each hypothesis. In general, the estimates, $\hat{\boldsymbol{s}}_{\mathcal{H}_{i}}^{(\mathrm{c})}$, is evaluated based on the periodic/anti-periodic properties of the layered ACO-OFDM and the DC bias is calculated using ML estimation.

The way the SCGs are defined leads to periodic/antiperiodic sequences at the outputs of the IFFTs at each singlelayer ACO-OFDM modulator [20]. For the $l$ th layer, the output of the IFFT includes $2^{l-1}$ identical sub-sequences, each being anti-periodic, i.e.

$$
\begin{aligned}
x_{l, k} & =-x_{l, k+N / 2^{l}}=x_{l, k+N / 2^{l-1}}=-x_{l, k+3 N / 2^{l}} \\
& =\cdots=x_{l, k+N-N / 2^{l-1}}=-x_{l, k+N-N / 2^{l}} .
\end{aligned}
$$

Therefore, the $k$ th and the $\left(k+N / 2^{l}\right)$ th, $0 \leq k \leq N / 2^{l}-1$, samples of the $L$-layer ACO-OFDM signal, $s_{k}^{(L)}$ and $s_{k+N / 2^{l}}^{(L)}$, given by (4), can be rewritten as

$$
s_{k}^{(L)}=\sum_{q=1}^{l-1} s_{q, k}+\frac{1}{2}\left(x_{l, k}+\left|x_{l, k}\right|\right)+\frac{1}{2} \sum_{q=l+1}^{L}\left(x_{q, k}+\left|x_{q, k}\right|\right)
$$

and

$s_{k+N / 2^{l}}^{(L)}=\sum_{q=1}^{l-1} s_{q, k+N / 2^{l}}+\frac{1}{2}\left(-x_{l, k}+\left|x_{l, k}\right|\right)+\frac{1}{2} \sum_{q=l+1}^{L}\left(x_{q, k}+\left|x_{q, k}\right|\right)$

respectively, resulting in the difference $s_{k}^{(L)}-s_{k+N / 2^{l}}^{(L)}$, given by

$s_{k}^{(L)}-s_{k+N / 2^{l}}^{(L)}=x_{l, k}+s_{1, k}+\cdots+s_{l-1, k}-s_{1, k+N / 2^{l}}-\cdots-s_{l-1, k+N / 2^{l}}$.

We apply the property of layered ACO-OFDM, (26), to deriving the estimate of the layered ACO-OFDM samples for each hypothesis. We denote the estimates of $\boldsymbol{x}_{l}, \boldsymbol{s}_{l}$ and $s^{(L)}$ conditioned on $\mathcal{H}_{i}$ by $\hat{\boldsymbol{x}}_{l}^{\mathcal{H}_{i}}=\left[\hat{x}_{l, 0}^{\mathcal{H}_{i}}, \cdots, \hat{x}_{l, N-1}^{\mathcal{H}_{i}}\right]^{T}, \hat{\boldsymbol{s}}_{l}^{\mathcal{H}_{i}}=$ $\left[\hat{s}_{l, 0}^{\mathcal{H}_{i}}, \cdots, \hat{s}_{l, N-1}^{\mathcal{H}_{i}}\right]^{T}$ and $\hat{\boldsymbol{s}}_{\mathcal{H}_{i}}^{(L)}=\left[\hat{s}_{0, \mathcal{H}_{i}}^{(L)}, \cdots, \hat{s}_{N-1, \mathcal{H}_{i}}^{(L)}\right]^{T}$, respectively. Then (23)-(26) also hold for the estimates. Conditioned on $\mathcal{H}_{i}$, the waveform of BCS is assumed to be $\boldsymbol{p}^{\mathcal{H}_{i}}$ in the received signal. Therefore, $u_{k}^{\mathcal{H}_{i}}=-p_{k} s_{k}^{(\mathrm{c})}+I_{\mathrm{c}}^{\prime}+w_{k}+A p_{k}-A p_{k}^{\mathcal{H}_{i}}$, can be rewritten as $u_{k}^{\mathcal{H}_{i}}=-p_{k}^{\mathcal{H}_{i}} \hat{s}_{k, \mathcal{H}_{i}}^{(\mathrm{c})}+I_{\mathrm{c}}^{\prime}+w_{k}$, where the real signal, $s_{k}^{(\mathrm{c})}$, is substituted by its estimate, $\hat{s}_{k, \mathcal{H}_{i}}^{(\mathrm{c})}$, under hypothesis $\mathcal{H}_{i}$. For the case where $\mathcal{H}_{i}$ is ture, i.e. $p_{k}=p_{k}^{\mathcal{H}_{i}}$, removing $A p_{k}^{\mathcal{H}_{i}}$ from the received signal will have no impact on the estimation of $s_{k}^{(\mathrm{c})}$, as the term removed is independent to $s_{k}^{(\mathrm{c})}$. However, for the cases where $\mathcal{H}_{i}$ is false, i.e. $p_{k} \neq p_{k}^{\mathcal{H}_{i}}$, the estimate, $\hat{s}_{k, \mathcal{H}_{i}}^{(\mathrm{c})}$, must compensate the difference between 
$-p_{k} s_{k}+A p_{k}-A p_{k}^{\mathcal{H}_{i}}$ and $-p_{k}^{\mathcal{H}_{i}} \hat{s}_{k, \mathcal{H}_{i}}^{(\mathrm{c})}$, and thus typically has large deviations from its real value.

We evaluate the difference between $u_{k+N / 2^{l}}^{\mathcal{H}_{i}}$ and $u_{k}^{\mathcal{H}_{i}}$ to yield

$$
\begin{gathered}
u_{k+N / 2^{l}}^{\mathcal{H}_{i}}-u_{k}^{\mathcal{H}_{i}}=p_{k}^{\mathcal{H}_{i}} \hat{s}_{k, \mathcal{H}_{i}}^{(\mathrm{c})}-p_{k+N / 2^{l}}^{\mathcal{H}_{i}} \hat{s}_{k+N / 2^{l}, \mathcal{H}_{i}}^{(\mathrm{c})}+w_{k+N / 2^{l}}-w_{k}, \\
1 \leq l \leq L, 0 \leq k \leq N / 2^{l}-1 .
\end{gathered}
$$

Comparing the right hand side (RHS) of (27) with the left hand side (LHS) of (26), we can see that if the vector $\boldsymbol{p}$ is $2^{L}$-fold periodical, i.e.

$$
p_{k}^{\mathcal{H}_{i}}=p_{k+N / 2^{L}}^{\mathcal{H}_{i}}=p_{k+N / 2^{L-1}}^{\mathcal{H}_{i}}=\cdots=p_{k+N-N / 2^{L}}^{\mathcal{H}_{i}},
$$

for all the hypotheses, (27) can be rewritten as

$$
u_{k+N / 2^{l}}^{\mathcal{H}_{i}}-u_{k}^{\mathcal{H}_{i}} \approx p_{k}^{\mathcal{H}_{i}}\left(\hat{s}_{k, \mathcal{H}_{i}}^{(L)}-\hat{s}_{k+N / 2^{l}, \mathcal{H}_{i}}^{(L)}\right) .
$$

The LHS and RHS of (29) approximately equal as a result of 1) the ignorance of the noise term, $w_{k+N / 2^{l}}-w_{k}$, and 2) assuming no signal clipping, i.e. $\hat{s}_{k, \mathcal{H}_{i}}^{(\mathrm{c})}=\hat{s}_{k, \mathcal{H}_{i}}^{(L)}$. By inserting (26) into the RHS of (29), the estimate, $\hat{x}_{l, k}^{\mathcal{H}_{i}}$, can be expressed as

$$
\begin{array}{r}
\hat{x}_{l, k}^{\mathcal{H}_{i}}=\frac{1}{p_{k}^{\mathcal{H}_{i}}}\left(u_{k+N / 2^{l}}^{\mathcal{H}_{i}}-u_{k}^{\mathcal{H}_{i}}\right)-\sum_{q=1}^{l-1} \hat{s}_{q, k}^{\mathcal{H}_{i}}+\sum_{q=1}^{l-1} \hat{s}_{q, k+N / 2^{l}}^{\mathcal{H}_{i}}, \\
1 \leq l \leq L, 0 \leq k \leq N / 2^{l}-1 .
\end{array}
$$

Eq. (30) implies that conditioned on hypothesis $\mathcal{H}_{i}$, the procedure for estimating the samples, $x_{l, k}$, is recursive in nature. As the estimates of the samples in the $l$ th layer require those in the previous layers, the estimation must be performed in an ascending order up to the $L$ th layer. In addition, it can be seen that only the first $N / 2^{l}$ samples in the lth layer are estimated using (30). The remaining samples will be recovered using the periodic/anti-periodic property, given by (23). Given the estimate, $\hat{x}_{l, k}^{\mathcal{H}_{i}}$, the estimate of the output of the $l$ th ACO-OFDM modulator, $\hat{s}_{l, k}^{\mathcal{H}_{i}}$, can be derived using (3), i.e. $\hat{s}_{l, k}^{\mathcal{H}_{i}}=\left(\hat{x}_{l, k}^{\mathcal{H}_{i}}+\left|\hat{x}_{l, k}^{\mathcal{H}_{i}}\right|\right) / 2$. Then, the estimates of the layered ACO-OFDM samples before clipping can be expressed as $\hat{s}_{k, \mathcal{H}_{i}}^{(L)}=\sum_{l=1}^{L} \hat{s}_{l, k}^{\mathcal{H}_{i}}$, leading to the estimates of the clipped samples, $\hat{\boldsymbol{s}}_{\mathcal{H}_{i}}^{(\mathrm{c})}$, in accordance with (7).

We estimate the DC bias, $I_{c}^{\prime}$, using an ML approach. Conditioned on hypothesis $\mathcal{H}_{i}$, the ML estimate of the DC bias, $\hat{I}_{\mathrm{c}}{ }^{\mathcal{H}_{i}}$, can be derived by taking the logarithm on (21), differentiating it with respect to $I_{\mathrm{c}}^{\prime}$, and forcing the result to zero to give

$$
\sum_{k=0}^{N-1}\left(\hat{I}_{\mathrm{c}}^{\mathcal{H}_{i}}-u_{k}^{\mathcal{H}_{i}}-p_{k}^{\mathcal{H}_{i}} \hat{s}_{k, \mathcal{H}_{i}}^{(\mathrm{c})}\right)=0
$$

Therefore the ML estimate of the DC bias can be expressed as

$$
\hat{I}_{\mathrm{c}}^{\mathcal{H}_{i}}=\frac{1}{N} \sum_{k=0}^{N-1}\left(p_{k}^{\mathcal{H}_{i}} \hat{s}_{k, \mathcal{H}_{i}}^{(\mathrm{c})}+u_{k}^{\mathcal{H}_{i}}\right)
$$

Combining the estimates given by (30) and (32) and the ML detector, (22), we summarize the demodulation procedure for FRPO-OFDM in Algorithm 1.

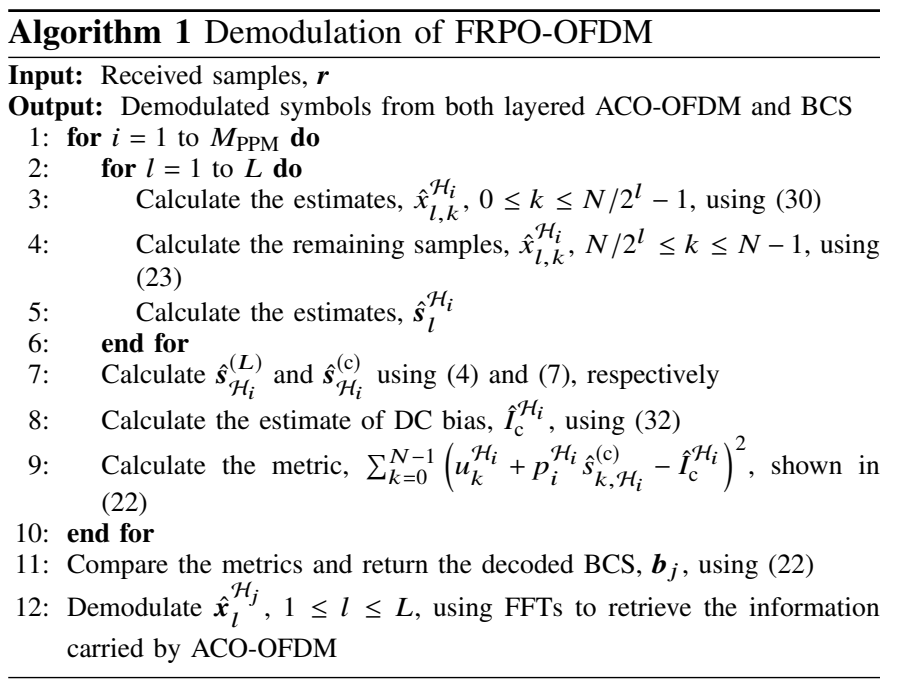

\section{B. Trade-Off Between Spectral Efficiency and Brightness Con- trol}

The estimator given by (30) imposes a $2^{L}$-fold periodical BCS. Thus, the codebook, $C^{\left(N, M_{\mathrm{PPM}}, c\right)}$, is no longer applicable from the signal detection point of view. Here, we introduce a modified codebook, $\tilde{C}^{\left(N, L, M_{\mathrm{PPM}}, c\right)}$, compatible with the proposed detector. The codeword, $\tilde{C}_{i}^{\left(N, L, M_{\mathrm{PPM}}, c\right)}, 0 \leq i \leq$ $M_{\mathrm{PPM}}-1$, in the new codebook consists of $2^{L}$ replicas of $C_{i}^{\left(N / 2^{L}, M_{\mathrm{PPM}}, c\right)}$, i.e.

$$
\tilde{C}_{i}^{\left(N, L, M_{\mathrm{PPM}}, c\right)}=\left[C_{i}^{\left(N / 2^{L}, M_{\mathrm{PPM}}, c\right)}, \cdots, C_{i}^{\left(N / 2^{L}, M_{\mathrm{PPM}}, c\right)}\right],
$$

where $C_{i}^{\left(N / 2^{L}, M_{\mathrm{PPM}}, c\right)}$ denotes the illustrative codeword consisting of $N / 2^{L}$ bits which are divided into $M_{\text {PPM }}$ groups with brightness level $c$.

As the codeword, $C_{i}^{\left(N / 2^{L}, M_{\mathrm{PPM}}, c\right)}$, consists of $N / 2^{L}$ bits, the order of PPM, $M_{\mathrm{PPM}}$, must not exceed $N / 2^{L}$, i.e. $M_{\mathrm{PPM}} \leq$ $N / 2^{L}$. Thus, the codebook given by (33) provides no more than $\left(N / 2^{L}-1\right)$ brightness levels between $I_{\mathrm{L}}+\bar{s}_{k}^{(\mathrm{c})}$ and $I_{\mathrm{H}}-\bar{s}_{k}^{(\mathrm{c})}$. This indicates the number of extra bits carried by the BCS and that of brightness steps decrease with increasing number of layers.

The information carried by an FRPO-OFDM signal comes from two sequences, the $L$-layer ACO-OFDM sequence and the BCS. For an L-layer ACO-OFDM, the number of independent bits loaded onto the subcarriers is given by [20]

$$
n_{\mathrm{ACO}-\mathrm{OFDM}}^{(L)}=\left(\frac{1}{4}+\frac{1}{8}+\cdots+\frac{1}{2^{L+1}}\right) N \log _{2} M_{\mathrm{OFDM}} .
$$

As $N / 2^{L}$ bits are included in $C_{i}^{\left(N / 2^{L}, M_{\mathrm{PPM}}, c\right)}$ in each codeword, the maximum number of the extra information bits carried by the BCS in the form of PPM is given by

$$
n_{\mathrm{BCS}}^{(L)}=\log _{2}\left(N / 2^{L}\right) \text {. }
$$

Therefore, the maximum number of bits carried by an FRPOOFDM signal can be expressed as

$$
n_{\mathrm{FRPO}-\mathrm{OFDM}}^{(L)}=\frac{1}{2}\left(1-\left(\frac{1}{2}\right)^{L}\right) N \log _{2} M_{\mathrm{OFDM}}+\log _{2}\left(N / 2^{L}\right) \text {. }
$$




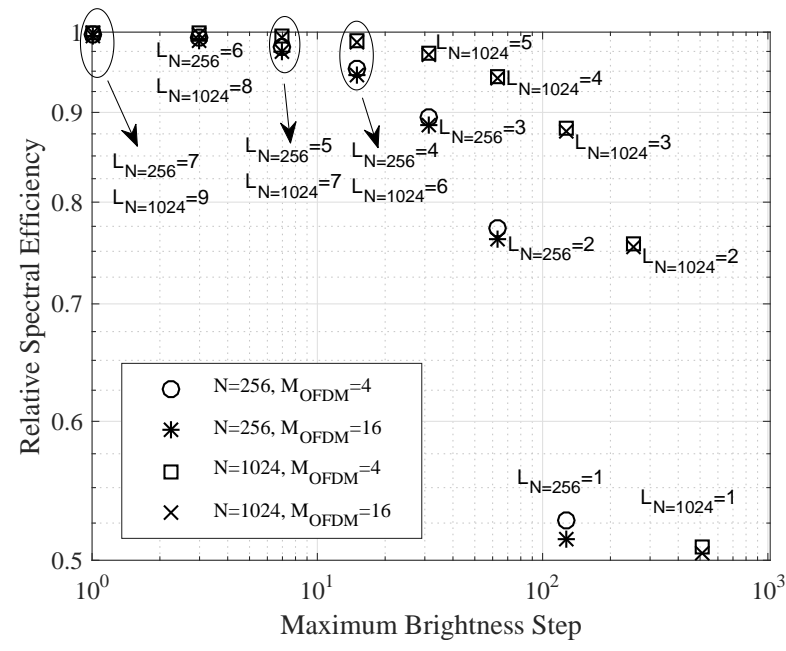

Fig. 4. Relative spectral efficiency versus maximum brightness step.

We normalize $n_{\text {FRPO-OFDM }}^{(L)}$ with the number of independent bits which can be carried by an original DCO-OFDM symbol, $n_{\text {DCO-OFDM }}$, as so far, it is known to have the highest spectral efficiency. The number, $n_{\text {DCO-OFDM }}$, can be expressed as

$$
n_{\mathrm{DCO}-\mathrm{OFDM}}=\left(\frac{N}{2}-1\right) \log _{2} M_{\mathrm{OFDM}} \approx\left(\frac{N}{2}\right) \log _{2} M_{\mathrm{OFDM}},
$$

leading to the relative spectral efficiency (RSE), $\eta_{L}$, given by

$$
\eta_{L}=\frac{n_{\mathrm{FRPO}-\mathrm{OFDM}}^{(L)}}{n_{\mathrm{DCO}-\mathrm{OFDM}}}=\left(1-\left(\frac{1}{2}\right)^{L}\right)+\left(\frac{2}{N}\right)\left[\frac{\left(\log _{2} N\right)-L}{\log _{2} M_{\mathrm{PPM}}}\right]
$$

Fig. 4 shows the RSE of the FRPO-OFDM signal as a function of the maximum brightness step supported, where $N=256$ and 1024 subcarriers are grouped into eight and ten layers/SCGs, respectively. As shown in the figure, the RSE drops with increasing step number. For single-layer/original ACO-OFDM which has only $N / 4$ subcarriers loaded with independent symbols, there are maximum $(N / 2-1)$ steps which potentially support very fine adjustment of the brightness level. The RSE in this case is slightly greater than that of the original RPO-OFDM [14] which equals 0.5 when ACO-OFDM is employed. For $\left(\log _{2} N\right)$-layer ACO-OFDM, however, the number of level supported drops to zero (not illustrated in the figure because of the use of the log-scale plot). This is because the corresponding BCS reduces to all one/zero sequence which does not support PPM. The figure indicates that the finest adjustment of brightness level and the highest spectral efficiency cannot be achieved at the same time. In practical scenario, the number of layer used in ACOOFDM must be selected to cope with the requirements on both dimming control and BER performance. From the perspective of dimming control, for an office room, a light switch with ten dimming steps may be sufficient. This, as shown in the figure, leads to over $90 \%$ of RSE if a four/six-layer ACO-OFDM with 256/1024 subcarriers is employed. From the viewpoint of communications, a preconfigured BER must be upheld. As the calculation of RSE does not take into account the decoding errors in the receiver [17][19][23], the number of layers the ACO-OFDM can employ may be further reduced, depending on the available power for the layered ACO-OFDM, the width of the dynamic range, and the noise power in the receiver.

\section{Computational Complexity}

In this section, we evaluate the numbers of (real) addition and multiplication that are additionally required to form and demodulate FRPO-OFDM symbols on top of the layered ACO-OFDM. In line with the evaluations for layered ACOOFDM [17] and for FFT/IFFT algorithms [22], we assume the size of IFFT/FFT is sufficiently large. Compared with the layered ACO-OFDM, the FRPO-OFDM signal shown in Fig. 1 indicates that the increase of complexity in the transmitter mainly comes from the element-wise multiplication and addition. The signal given by (6) shows that two extra additions and one extra multiplication are required to form an FRPO-OFDM sample using a layered ACO-OFDM one. Thus, for an OFDM symbol with $N$ samples, the extra number of additions and multiplications are given by $2 N$ and $N$, respectively.

Compared with a layered ACO-OFDM receiver, the complexity increased in the FRPO-OFDM receiver is mainly caused by the estimator and detector, i.e. steps 3, 7, 8 and 9 of Algorithm $1^{4}$. The numbers of additions and multiplications required for each step are summarized in Table II. See Appendix B for the derivations. As there are $M_{\mathrm{PPM}}$ hypotheses for the BCS, these steps are performed $M_{\mathrm{PPM}}$ times followed by a decision made in favour of the smallest metric (step 11). From Table II, we can see that the overall numbers of additions and multiplications, $N_{\text {add }}$ and $N_{\text {mul }}$, required to go through all the hypotheses are approximately given by

$$
N_{\text {add }}=(7+L) M_{\mathrm{PPM}} N \text {, and } N_{\text {mul }}=4 M_{\mathrm{PPM}} N \text {, }
$$

respectively. These numbers indicate that for a given layered ACO-OFDM modulator (fixed $L$ ), the extra computation load in the receiver depends on the value for $M_{\mathrm{PPM}}$.

The trade-off between the RSE and brightness control shows that for a given $L$, the order of PPM, $M_{\mathrm{PPM}}$, can take integer values up to $N / 2^{L}$. Inserting these values into (39), we can see that the numbers required are proportional to either $N$ or $N^{2}$, depending on the relationship between $M_{\text {PPM }}$ and $N$. For a conservative configuration in which only the requirement on dimming control is to be met, the value for $M_{\mathrm{PPM}}$ will be dependent on the number of the required brightness steps, but typically independent of $N$. The resulting computational complexity is therefore proportional to $N$. Alternatively, the maximum data rate and/or the finest brightness control can be achieved by the BCS when a greedy configuration is implemented. In this configuration, the maximum value, $N / 2^{L}$, is taken by $M_{\text {PPM }}$. This implies that the order of PPM becomes dependent on $N$, and thus results in the computational complexity proportional to $N^{2}$.

The computation load of a layered ACO-OFDM receiver is proportional to $N \log _{2} N$ [17]. This indicates that the additional

\footnotetext{
${ }^{4}$ We assume no additions or multiplications are performed to convert $\hat{x}_{l, k}^{\mathcal{H}_{i}}$ to $\hat{s}_{l, k}^{\mathcal{H}_{i}}$, as only the signs of $\hat{x}_{l, k}^{\mathcal{H}_{i}}$ need to be identified in the conversion.
} 
computation caused by the informative BCS relative to the layered ACO-OFDM receiver depends on the configuration of BCS. For greedy configuration, the numbers of additions and multiplications which are proportional to $N^{2}$ will significantly increase the computation load on top of a layered ACO-OFDM receiver. For a conservative configuration, on the contrary, the computation load for a layered ACO-OFDM still dominates. Thus the extra complexity caused by the informative BCS will not be significant.

\section{Simulation Results}

We present the simulation results for both dimming and BER performance that can be achieved by FRPO-OFDM. The simulations are configured using the following parameters:

1) Layered ACO-OFDM: Unless specified otherwise, the $L$ layer ACO-OFDM modulator modulates 4-QAM symbols onto $N=256$ subcarriers.

2) $B C S$ : Unless specified otherwise, 16-PPM is employed to provide 15 brightness steps.

3) FRPO-OFDM Signal: The dynamic range of the samples is normalized between $I_{\mathrm{L}}=0$ and $I_{\mathrm{H}}=1$, and is assumed identical to that of the LED luminaire. Then the resulting currents, power and energy are normalized values with respect to this range. The bandwidth of the signal, $W_{\mathrm{s}}$, is set to be $2 \mathrm{MHz}$. Here, we assume that the increase of the dynamic range due to DAC is negligible and thus that the dynamic range of the samples equals that of the resulting analogue signals.

4) LED: The modulation bandwidth of the LED is assumed greater than $2 \mathrm{MHz}$, so the electrical-to-optical conversion factor, $\alpha_{\mathrm{eo}}$, in the LED is a constant on all the subcarriers.

5) Photodetector: Without loss of generality, the responsivity of the photodetector is assumed equal to $1 / \alpha_{\mathrm{eo}}$ [26]. Then, the received signal can be expressed as (20).

6) Noise: The shot noise after being anti-aliasing filtered is assumed to have a bandwidth identical to that of the wanted signal. Its normalized power, $\sigma_{\mathrm{w}}^{2}$, is given by 0.001 . This value is selected to illustrate the full behaviour of BER against $E_{\mathrm{b} \text {,elec }} / N_{0}$ from 0 to $26 \mathrm{~dB}$. Here, the single-sided power spectral density of the noise, $N_{0}$, is evaluated as $\sigma_{\mathrm{w}}^{2} / W_{\mathrm{s}}$.

\section{A. Measured and Perceived Brightness}

As shown in (8)-(19), the MBL of an FRPO-OFDM signal is a function of the group number, $M_{\mathrm{PPM}}$, in the BCS, the energy of symbols, $\epsilon$, the number of layer used in the ACO-OFDM and the brightness step, $c$. Fig. 5 illustrates the interactions between these parameters and the MBL. We consider the single- and double-layer ACO-OFDM signals, each clipped with probability of 0.01 and 0.1 , respectively. Using the PDF given by (15) with $\sigma_{1}^{2}=\epsilon / 2$, these, for single layer ACOOFDM, correspond to $\epsilon=0.37$, for the probability of 0.01 and 1.21 for 0.1 , and lead to the expected value, $\bar{s}_{k}^{(c)}=0.17$ and 0.27 , respectively. When 4-QAM symbols are loaded onto the subcarriers, the resulting values for $E_{\mathrm{b} \text {,elec }} / N_{0}$ are given by $19.67 \mathrm{~dB}$ (for the probability of 0.01 ) and $27.71 \mathrm{~dB}$ (for

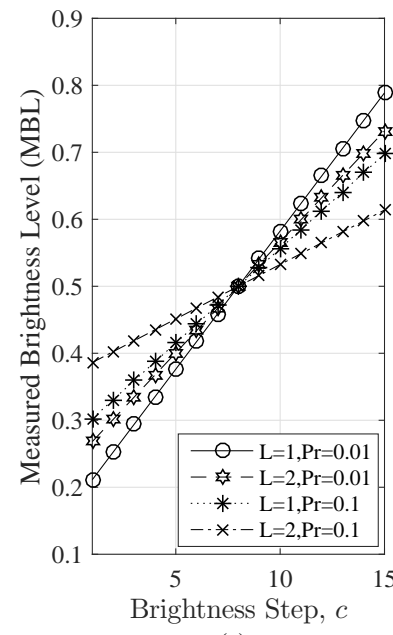

(a)

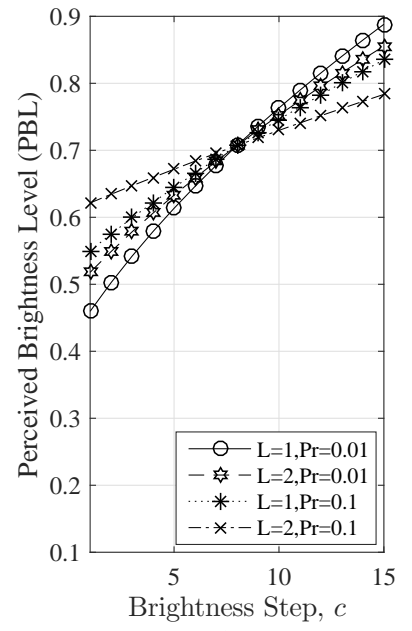

(b)
Fig. 5. Measured and perceived brightness level.

the probability of 0.1 ), respectively. For double layer ACOOFDM, bi-section method is applied, returning $\epsilon=0.24$ for the probability of 0.01 and 0.7 for 0.1 , which correspond to $\bar{s}_{k}^{(c)}=0.24$ and 0.37 , respectively. The resulting values for $E_{\mathrm{b} \text {,elec }} / N_{0}$ are given by $18.92 \mathrm{~dB}$ (for the probability of 0.01 ) and $23.57 \mathrm{~dB}$ (for the probability of 0.1 ), respectively.

Fig. 5(a) shows the MBL as a function of the brightness step. From this figure, we can see that the MBL increases linearly with the step, $c$. This is desirable in the lighting industry. Fig. 5(b) shows the PBL. As shown in the figure, because of the nonlinear relationship between the MBL and PBL which is caused by the adjustment of pupil size, the light perceived is always brighter than that measured. It can be seen that the impact of the energy, $\epsilon$, and that of the number of layer on the MBL are reflected through the overall range of the brightness. As shown in Fig. 5(a), single layer ACOOFDM with the probability of clipping at 0.01 leads to the widest brightness range from 0.2 to 0.8 , whereas double layer with probability of 0.1 , the narrowest, only from 0.4 to 0.6 . This can be explained as follows. As shown in (9), the overall range of brightness depends on the expected value, $\bar{s}_{k}^{(\mathrm{c})}$, which tends to lift up the off state and to close down the on state of the BCS. Therefore, a low value for it will lead to a wide range of brightness, whereas a high value, a narrow range. The expected value depends on the PDF of the ACO-OFDM signal which is a function of $\epsilon$ and the number of layer, $L$ (see (19)). As the continuous component of the PDF is an increasing function of the number of layer (see Fig. 3) and symbol energy (because of the clipped Gaussian distributed output of each modulator), the expected value increases with increasing number of layer and $\epsilon$. Hence, as shown in Fig. 5, a smaller number of layer/probability of clipping produces a wider range of brightness.

\section{B. BER Performance}

Apart from the impact on illumination, the energy, $\epsilon$, also has a complex impact on the BERs of the BCS and ACOOFDM signal. First, for the demodulation of BCS, as the ML 
TABLE II

COMPUTATIONAL COMPLEXITY

\begin{tabular}{|c|c|c|c|c|c|}
\hline & Step 3 & Step 7 & Step 8 & Step 9 & Overall \\
\hline Add. & $\left(3-(3+2 L) / 2^{L}\right) N$ & $N(L-1)$ & $2 N-1$ & $3 N-1$ & $\left(7+L-3 / 2^{L}-L / 2^{L-1}\right) N-2$ \\
\hline Mul. & $\left(1-(1 / 2)^{L}\right) N$ & 0 & $N$ & $2 N$ & $\left(4-(1 / 2)^{L}\right) N$ \\
\hline
\end{tabular}

detector, (22), takes the ACO-OFDM sequence as a nuisance parameter, the increase of $\epsilon$ leading to the increase of the power of the ACO-OFDM sequence will result in degrading BERs. Second, because of the fixed dynamic range of the samples/LED and the decoding errors of the BCS which cause the error propagation, the way the BER of ACO-OFDM varies as a function of the energy will be very different from that published in conventional ACO-OFDM papers [5]. For small energy for which the signal clipping and the decoding errors are negligible, the increase of energy will help combat the noise in the detector and estimator, leading to improved BER for the ACO-OFDM signal. However, as the energy increases continuously, the decoding errors start to take effect and the frequent signal clippings causing SNR degradation [27] become dominating. Thus, the estimator gradually loses precision, resulting in degrading BERs for both sequences. Because of the relationship between $P_{\text {elec }}$ and $E_{\mathrm{b} \text {,elec }}$ which denote the electrical power and the energy per bit before clipping, respectively, the value for $E_{\mathrm{b} \text {,elec }} / N_{0}$ is proportional to $\epsilon$. Thus, the way BERs vary with $E_{\mathrm{b} \text {,elec }} / N_{0}$ is identical to that they vary with $\epsilon$.

Fig. 6 shows the BERs versus $E_{\mathrm{b}, \text { elec }} / N_{0}$ for the $M_{\mathrm{PPM}^{-}}$ PPM and layered ACO-OFDM signals where 16-PPM is used in the BCS combined with single-, double- and triple-layer ACO-OFDM signals. The brightness step, $c$, is configured at 1 and 8, respectively. As shown in the figure, the BER of PPM grows with increasing energy, whereas the BER for ACOOFDM first drops and then increases with increasing $\epsilon$. This verifies the analysis about the impact of $\epsilon$ on the BER. It can be seen that for $E_{\mathrm{b} \text {,elec }} / N_{0}$ ranging from $0 \mathrm{~dB}$ to $16 \mathrm{~dB}$ where signal clipping is not dominating, the BER of ACO-OFDM depends only on the number of layer, but is independent of the brightness step. In this range, there are zero errors found from the $\mathrm{BCS}^{5}$. These are desirable for dimming compatible VLC in which the BER is required not to be affected by dimming control. For the range above $19 \mathrm{~dB}$, however, the clipping noise and the resulting decoding errors of BCS become dominating and thus the BER degrades with increasing energy for both the BCS and the layered ACO-OFDM. Therefore, in line with the original optical OFDM, this range should be avoided in the system design. In this range, we can see that the eighth step of brightness causes slightly lower BER than the first step for both the BCS and the layered ACO-OFDM. This is caused by the different distance distributions of the two BCS codebooks. For the first brightness step, a codeword in $\tilde{C}^{(256, L, 16,1)}, L=1,2,3$, consistently distinguishes with other codewords by two groups (i.e. 32 bits). However, for the eighth

\footnotetext{
${ }^{5}$ In fact, zero errors are returned from the simulation for the BCS combined with single-layer ACO-OFDM in the whole range considered.
}

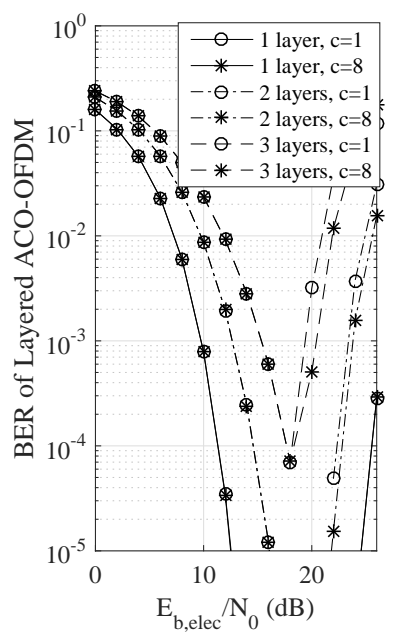

(a)

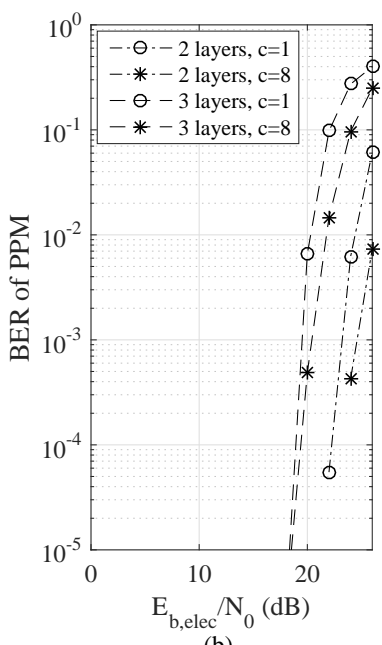

(b)
Fig. 6. BERs of the layered ACO-OFDM and BCS.

step, the distance is not a constant, and is minimum two groups. For the codebook, $\tilde{C}^{(256, L, 16,8)}, L=1,2,3$, used in the simulation, we can see that given a codeword, the distance between this codeword and the others can be 2, 4, 6, 8, 10, 12,14 and 16 groups which are either higher than or equal to that for the first step. Therefore, the eighth step will lead to lower BERs of BCS than the first step as shown in Fig. 6(b). As less error propagations occur for the eighth step, the resulting BERs of layered ACO-OFDM are slightly lower than those for the first step as shown in Fig. 6(a).

Fig. 7 shows the constellation diagrams of the double-layer ACO-OFDM obtained using the same configurations with those for Fig. 6. The dimming level is set at the first step, i.e. $c=1$. Two values for $E_{\mathrm{b} \text {,elec }} / N_{0}$, equalling $16 \mathrm{~dB}$ and $24 \mathrm{~dB}$, respectively, are studied. As shown in Fig. $6, E_{\mathrm{b} \text {,elec }} / N_{0}=16$ $\mathrm{dB}$ corresponds to a low BER scenario, whereas $24 \mathrm{~dB}$, high BERs for both ACO-OFDM and BCS. From the figure, we can see that the constellation diagrams for $E_{\mathrm{b}, \text { elec }} / N_{0}=16 \mathrm{~dB}$, shown in Fig. 7(a) and (b), are consistent with those of original OFDM at high signal-to-noise ratio (SNR). This is because in this case, the signal clipping does not occur frequently and the BCS can be detected correctly with extremely high probability. Thus the informative BCS has negligible impact on the demodulation of ACO-OFDM. As the demodulation process given by (30) indicates that the estimation of the signals in the $l$ th layer depends on those in the previous layers, the estimation errors will accumulate, resulting in increasing noise power as shown in Fig. 7(a) and (b). Fig. 7(c) and (d) plot the constellation diagrams for $E_{\mathrm{b} \text {,elec }} / N_{0}=24 \mathrm{~dB}$. As signal clipping (of ACO-OFDM) and the detection errors (of 


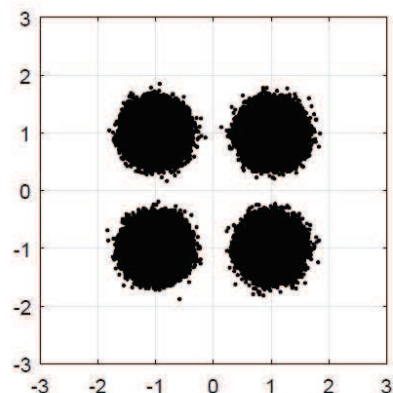

(a)

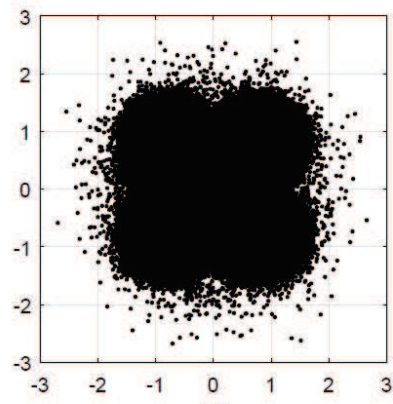

(c)

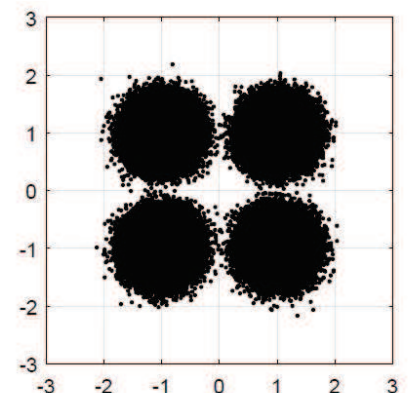

(b)

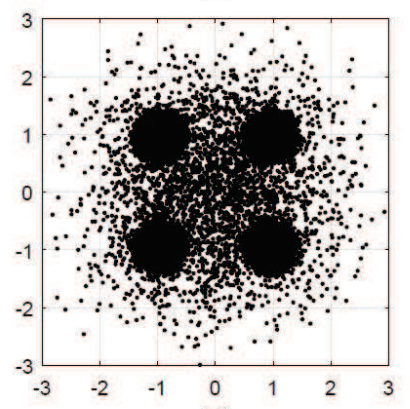

(d)
Fig. 7. Constellation plots, (a) 1st layer with $E_{\mathrm{b} \text {,elec }} / N_{0}=16 \mathrm{~dB}, \mathrm{BER}=0$, (b) 2nd layer with $E_{\mathrm{b} \text {, elec }} / N_{0}=16 \mathrm{~dB}, \mathrm{BER}=3.125 \times 10^{-5}$, (c) 1 st layer with $E_{\mathrm{b}, \text { elec }} / N_{0}=24 \mathrm{~dB}, \mathrm{BER}=4.1 \times 10^{-3}$, (d) 2 nd layer with $E_{\mathrm{b} \text {, elec }} / N_{0}=24$ $\mathrm{dB}, \mathrm{BER}=2.1 \times 10^{-3}$.

BCS) occur frequently, both diagrams are superpositions of those of clipped OFDM signals and scattered dots randomly distributed. From both diagrams, we can see that the detection error causes the scatter plots behaving like noise with zero mean. This is because when it occurs, as shown in (30), there will be $2 N / M_{\mathrm{PPM}}=32$ out of $N=256$ samples estimated with large estimation error. From the excessive shrinkage of constellation, it can be seen that the ACO-OFDM signal in the first layer is significantly distorted by clippings [27]. We can also see that unlike the first layer, signal clipping does not have a major impact on the second layer. We attribute this to 1) the much lower electrical power of $s_{2}$ than that of $s_{1}$, and 2) the order of estimation which imposes a direct impact of clipping on the first layer but an indirect one on the subsequent layers.

For a close look at the impact of error propagation, we assume an incorrect decision is made in favour of the $i$ th codeword of codebook $\tilde{C}^{(256,2,16,1)}$, whereas the $j$ th codeword is transmitted. Then the estimates conditioned on both the hypotheses can be expressed as

$$
\begin{aligned}
\hat{x}_{1, k}^{\mathcal{H}_{i}} & =\frac{1}{p_{k}^{\mathcal{H}_{i}}}\left(r_{k+N / 2}-r_{k}\right), \\
\hat{x}_{1, k}^{\mathcal{H}_{j}} & =\frac{1}{p_{k}^{\mathcal{H}_{j}}}\left(r_{k+N / 2}-r_{k}\right), \\
\hat{x}_{2, k}^{\mathcal{H}_{i}} & =\frac{1}{p_{k}^{\mathcal{H}_{i}}}\left(r_{k+N / 4}-r_{k}\right)-\hat{s}_{1, k}^{\mathcal{H}_{i}}+\hat{s}_{1, k+N / 4}^{\mathcal{H}_{i}}, \\
\hat{x}_{2, k}^{\mathcal{H}_{j}} & =\frac{1}{p_{k}^{\mathcal{H}_{j}}}\left(r_{k+N / 4}-r_{k}\right)-\hat{s}_{1, k}^{\mathcal{H}_{j}}+\hat{s}_{1, k+N / 4}^{\mathcal{H}_{j}} .
\end{aligned}
$$

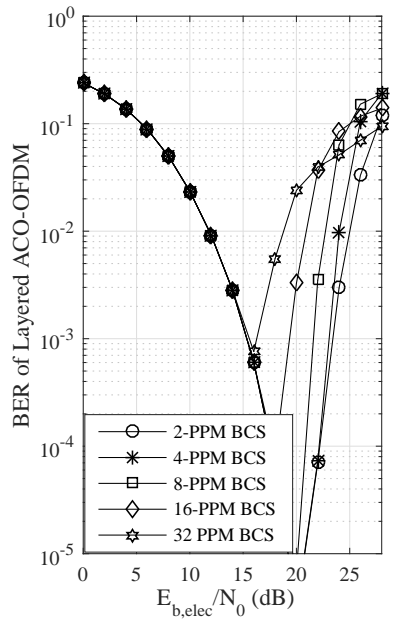

(a)

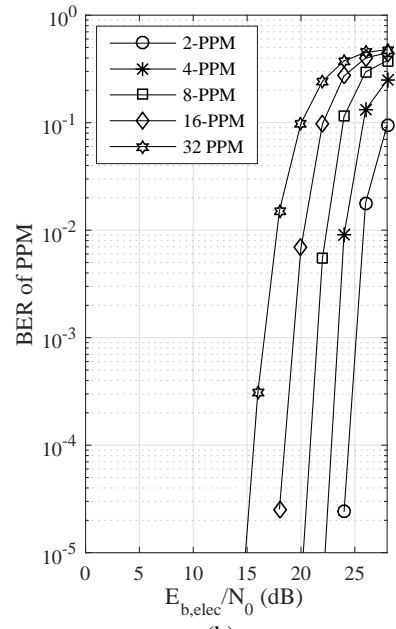

(b)
Fig. 8. BERs of the three-layer ACO-OFDM and BCS using 2, 4, 8, 16 and 32-PPM.

From (40), we can see that the estimates of the first layer, $\hat{x}_{1, k}^{\mathcal{H}_{i}}$ and $\hat{x}_{1, k}^{\mathcal{H}_{j}}$, are related by

$$
\hat{x}_{1, k}^{\mathcal{H}_{i}}=p_{k}^{\mathcal{H}_{i}} p_{k}^{\mathcal{H}_{j}} \hat{x}_{1, k}^{\mathcal{H}_{j}}=\left\{\begin{array}{l}
\hat{x}_{1, k}^{\mathcal{H}_{j}}, \quad \text { if } p_{k}^{\mathcal{H}_{i}}=p_{k}^{\mathcal{H}_{j}} \\
-\hat{\mathcal{H}}_{1, k}, \quad \text { if } p_{k}^{\mathcal{H}_{i}}=-p_{k}^{\mathcal{H}_{j}}
\end{array},\right.
$$

where $\hat{x}_{1, k}^{\mathcal{H}_{i}}, 0 \leq k \leq N-1$, corresponds to the samples subject to error propagation, and $\hat{x}_{1, k}^{\mathcal{H}_{j}}, 0 \leq k \leq N-1$, the samples to be demodulated if a correct decision is made. As any two codewords in $\tilde{C}^{(256,2,16,1)}$ distinguish by $2 N / M_{\mathrm{PPM}}=32$ bits, 32 samples in $\hat{\boldsymbol{x}}_{1}^{\mathcal{H}_{i}}$ have opposite signs to their corresponding ones in $\hat{\boldsymbol{x}}_{1}^{\mathcal{H}_{j}}$. Denoting the FFT of $\hat{\boldsymbol{x}}_{1}^{\mathcal{H}_{i}}$ and $\hat{\boldsymbol{x}}_{1}^{\mathcal{H}_{j}}$ by $\hat{\boldsymbol{X}}_{1}^{\mathcal{H}_{i}}$ and $\hat{\boldsymbol{X}}_{1}^{\mathcal{H}_{j}}$, respectively, they are related by

$$
\hat{\boldsymbol{X}}_{1}^{\mathcal{H}_{i}}=\boldsymbol{P}_{i, j} \hat{\boldsymbol{X}}_{1}^{\mathcal{H}_{j}}
$$

where $\boldsymbol{P}_{i, j}$ denotes the circulant (convolution) matrix formed by the FFT of $\boldsymbol{p}^{\mathcal{H}_{i}} \otimes \boldsymbol{p}^{\mathcal{H}_{j}}$, where $\otimes$ represents the elementwise multiplication. Eq. (42) implies that the incorrect decision results in inter-carrier interference in the first layer.

Eq. (40) indicates that the estimates of the second layer under both hypotheses are related by

$$
\begin{aligned}
\hat{x}_{2, k}^{\mathcal{H}_{i}} & =p_{k}^{\mathcal{H}_{i}} p_{k}^{\mathcal{H}_{j}}\left(\hat{x}_{2, k}^{\mathcal{H}_{j}}+\hat{s}_{1, k}^{\mathcal{H}_{j}}-\hat{s}_{1, k+N / 4}^{\mathcal{H}_{j}}\right)-\hat{s}_{1, k}^{\mathcal{H}_{i}}+\hat{s}_{1, k+N / 4}^{\mathcal{H}_{i}} \\
& =\left\{\begin{array}{lc}
\hat{x}_{2, k}^{\mathcal{H}_{j},} \quad \text { if } p_{k}^{\mathcal{H}_{i}}=p_{k}^{\mathcal{H}_{j}} \\
-\hat{x}_{2, k}^{\mathcal{H}_{j}}-\left|\hat{x}_{1, k}^{\mathcal{H}_{j}}\right|+\left|\hat{x}_{1, k+N / 4}^{\mathcal{H}_{j}}\right|, \quad \text { if } p_{k}^{\mathcal{H}_{i}}=-p_{k}^{\mathcal{H}_{j}} .
\end{array}\right.
\end{aligned}
$$

It can be seen that similar with the first layer, the incorrect decision only affects 32 samples corresponding to those with $p_{k}^{\mathcal{H}_{i}}=-p_{k}^{\mathcal{H}_{j}}$. The affected samples comprise two components: a flipped $\hat{x}_{2, k}^{\mathcal{H}_{j}}$ that causes inter-carrier interference and a residue-interference term, $-\left|\hat{x}_{1, k}^{\mathcal{H}_{j}}\right|+\left|\hat{x}_{1, k+N / 4}^{\mathcal{H}_{j}}\right|$, from the first layer. Therefore, when $\hat{\boldsymbol{x}}_{2}^{\mathcal{H}_{i}}$ is used to retrieve the information, the demodulation will be subject to both inter-carrier and interlayer interference. 


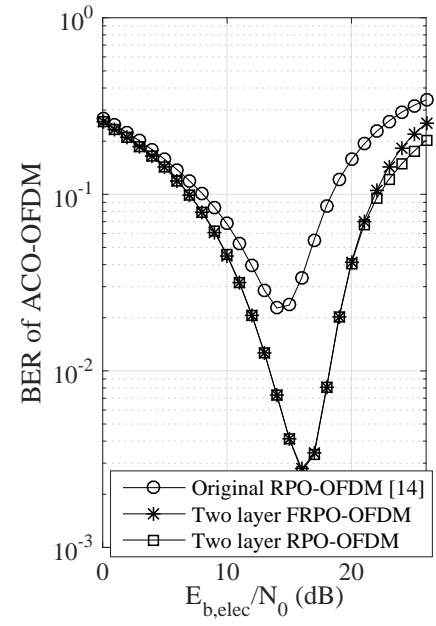

(a)

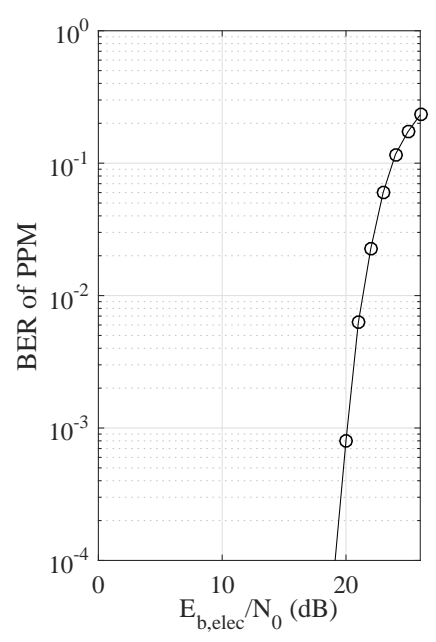

(b)
Fig. 9. Comparison between FRPO-OFDM, original RPO-OFDM and twolayer RPO-OFDM.

Fig. 8 shows the BERs of FRPO-OFDM which is generated by triple-layer ACO-OFDM and a BCS using $M_{\mathrm{PPM}} \mathrm{PPM}$. Because of the periodicity of the BCS, up to $\log _{2}\left(N / 2^{3}\right)=5$ bits can be transmitted by the BCS, indicating that the candidate forms of BCS include 2, 4, 8, 16 and 32-PPM. It can be seen that the BER of the BCS degrades with increasing bit transmitted. This is because the BER of $M$-PPM depends on the minimum Hamming distance of the codebook. As the minimum Hamming distance of the modified codebook equals $2 N / M_{\text {PPM }}$ bits (two groups), the increase of the modulation order, $M_{\mathrm{PPM}}$, will decrease this distance, resulting in degrading BERs. We can also see that consistent BERs of ACO-OFDM can be achieved regardless of the order of PPM when the value for $E_{\mathrm{b} \text {,elec }} / N_{0}$ is not greater than $16 \mathrm{~dB}$. Above this, the error propagation and signal clipping will start to take effect and lead to growing BERs.

\section{Comparison with Original RPO-OFDM}

In this section, we compare the BERs achieved by the ACOOFDM component of FRPO-OFDM and by the original RPOOFDM [14]. The original RPO-OFDM employs a single layer ACO-OFDM as described in [14] whereas the FRPO-OFDM combines a double-layer ACO-OFDM with a BCS in the form of 16-PPM. Identical spectral efficiency is assumed for the ACO-OFDM signals in both dimming compatible schemes, i.e. the relationship, (44), holds:

$$
\left(\frac{N}{4}+\frac{N}{8}\right) \log _{2} M_{\mathrm{OFDM}}=\left(\frac{N}{4}\right) \log _{2} M_{\mathrm{RPO}}^{(1)} .
$$

where $M_{\mathrm{RPO}}^{(1)}$ denotes the order of the QAM symbols loaded onto the original RPO-OFDM signals. As a direct extension, double-layer ACO-OFDM can be employed in RPO-OFDM to improve the spectral efficiency. This extension is referred to the two-layer RPO-OFDM which loads $M_{\mathrm{RPO}}^{(2)}-\mathrm{QAM}$ symbols onto its subcarriers.

Fig. 9(a) shows the BERs of the ACO-OFDM components in the three schemes where $M_{\mathrm{OFDM}}, M_{\mathrm{RPO}}^{(1)}$ and $M_{\mathrm{RPO}}^{(2)}$ are given by 16, 64 and 16, respectively. We assume that for the original and two-layer RPO-OFDM, the on and off states can be always identified correctly and thus ignore the resulting error. Therefore, the BER of the original RPO-OFDM scheme is equivalent to that of an ACO-OFDM signal with signal clipping to its peaks at $2 A$. For FRPO-OFDM, we employ the estimator and detector given by (30) and (22) to demodulate the information carried by the layered ACO-OFDM. For the two-layer RPOOFDM, the samples are recovered using the approach given by (26) and the periodic/anti-periodic property, (23), to ensure a similar receiver structure with FRPO-OFDM. As shown in the figure, the two double-layer OFDM schemes outperform the original RPO-OFDM. This is because the original RPOOFDM employs a larger constellation size which dominates the BER performance. As shown in (44), the number of bit per symbol for RPO-OFDM must be 1.5 times of those for the double-layer OFDM schemes. This implies that when the double-layer schemes use a constellation size greater than 64, original RPO-OFDM will have to employ impractically large constellation size (512-QAM for RPO-OFDM), resulting in extremely high BERs. We can also see that the two doublelayer schemes have the same BER performance until the value for $E_{\mathrm{b} \text {,elec }} / N_{0}$ grows up to $22 \mathrm{~dB}$. This confirms that although being subject to error propagation, the informative BCS of the FRPO-OFDM has negligible impact on the demodulation of the ACO-OFDM component. As shown in Fig. 9(b), the BER of the BCS is extremely low for a wide range from 0 to $19 \mathrm{~dB}$. This demonstrates that a second data stream with extremely high reliability can be provided by the proposed FRPO-OFDM in addition to the high speed ACO-OFDM sequence.

\section{CONCLUSION}

We have studied FRPO-OFDM for indoor dimming compatible VLC. The new signal format is derived as a combination of a multi-layer ACO-OFDM sequence with an informationcarrying $\mathrm{BCS}$ in the form of $M$-PPM. We show that the new OFDM scheme can fully utilize an LED's dynamic range, avoiding significant nonlinear distortion in adjusting the brightness level. The achievable brightness levels are derived as a function of the applied sequences. It is shown that the range of brightness levels drops with increasing symbol energy and increasing number of layers. The detector for FRPOOFDM is developed, which can recover information from both the sequences. A trade-off between spectral efficiency and maximum brightness steps is presented when this detector is to be implemented. We show that although the finest brightness control and the highest spectral efficiency cannot be achieved simultaneously, a dimming compatible VLC system providing more than 10 brightness levels with 90 percent of RSE is achievable, which copes with most indoor scenarios. The simulation results demonstrate that the desirable linear relationship exists between the MBL and the brightness step. The simulated BER shows that when signal clipping is not dominant, the use of the information-carrying BCS provides a highly reliable data stream, but has negligible impact on the BER of the ACO-OFDM signal. 


\section{APPENDIX A}

\section{DERIVATION OF THE PROPOSED APPROACH}

Because of the randomness, the output of the $L$-layer ACOOFDM modulator can be expressed, in terms of that of an $(L-1)$-layer ACO-OFDM modulator and that of the $L$ th layer, as

$$
s_{k}^{(L)}=\left\{\begin{array}{ll}
0, & \text { if } s_{k}^{(L-1)}=0, s_{L, k}=0 \\
s_{L, k}, & \text { if } s_{k}^{(L-1)}=0, s_{L, k}>0 \\
s_{k}^{(L-1)}, & \text { if } s_{k}^{(L-1)}>0, s_{L, k}=0 \\
s_{k}^{(L-1)}+s_{L, k}, & \text { if } s_{k}^{(L-1)}>0, s_{L, k}>0
\end{array},\right.
$$

with the probabilities corresponding to each case given by

$$
\begin{aligned}
& P\left(s_{k}^{(L)}=0\right)=2^{-L}, \quad P\left(s_{k}^{(L)}=s_{L, k}\right)=2^{-L}, \\
& P\left(s_{k}^{(L)}=s_{k}^{(L-1)}\right)=0.5-2^{-L}, \\
& P\left(s_{k}^{(L)}=s_{k}^{(L-1)}+s_{L, k}\right)=0.5-2^{-L} .
\end{aligned}
$$

Therefore, using the law of total probability [28], the PDF of $s_{k}$ can be expressed as (11).

Using the Gaussian-distribution approximation, the conditional PDF of the output of the $L$ th single layer modulator can be expressed as (12)

As the output of an $(L-1)$-layer ACO-OFDM modulator equals zero with a probability of $2^{-(L-1)}$, the conditional probability, $f_{s_{k}^{(L-1)}}\left(s \mid s_{k}^{(L-1)}>0\right)$, can be rewritten as (13).

The conditional PDF, $f_{s_{k}^{(L-1)}+s_{L, k}}\left(s \mid s_{k}^{(L-1)}>0, s_{L, k}>0\right)$, can be derived using a linear transformation between $\left[s_{k}^{(L-1)}, s_{L, k}\right]^{T}$ and $\left[\tilde{s}_{k}^{(L)}, z\right]^{T}$ where $\tilde{s}_{k}^{(L)}=s_{k}^{(L-1)}+s_{L, k}$ and $z=s_{L, k}$, leading to the corresponding Jacobian , $|\boldsymbol{J}|$, equalling one. Therefore the joint PDF of $\left[\tilde{s}_{k}^{(L)}, z\right]^{T}$ can be expressed as [29]

$$
\begin{aligned}
& f_{\tilde{s}_{k}^{(L)}, z}\left(s, z \mid s_{k}^{(L-1)}>0, s_{L, k}>0\right) \\
= & f_{s_{k}^{(L-1)}, s_{L, k}}\left(s-z, z \mid s_{k}^{(L-1)}>0, s_{L, k}>0\right)|\boldsymbol{J}| \\
= & f_{s_{k}^{(L-1)}}\left(s-z \mid s_{k}^{(L-1)}>0\right) f_{s_{L, k}}\left(z \mid s_{L, k}>0\right) \\
= & \frac{1}{1-2^{-(L-1)}} f_{s_{k}^{(L-1)}}(s-z) f_{s_{L, k}}\left(z \mid s_{L, k}>0\right),
\end{aligned}
$$

leading to the PDF of $\tilde{s}_{k}^{(L)}$ given by (14).

\section{APPENDIX B}

\section{DERIVATION OF COMPUTATIONAL COMPLEXITY}

First, we study the computational complexity of step 3 of Algorithm 1. As shown in (30), the numbers of additions and multiplications required to estimate a sample depend on the layer to be estimated. For the $l$ th layer, $(2 l-1)$ additions and one multiplication are performed for each sample. This leads to $\left(N / 2^{l}\right)(2 l-1)$ additions and $\left(N / 2^{l}\right)$ multiplications for all the samples in the $l$ th layer. Thus, the overall number of additions, $N_{\hat{x}}^{\text {add }}$, and that of multiplications, $N_{\hat{x}}^{\text {mul }}$, required to calculate the estimates, $\hat{x}_{l, k}^{\mathcal{H}_{i}}, 1 \leq l \leq L, 0 \leq k \leq N / 2^{l}-1$, can be expressed as

$$
\begin{aligned}
& N_{\hat{x}}^{\mathrm{add}}=\sum_{l=1}^{L}\left(\frac{N}{2^{l}}\right)(2 l-1)=\left(3-(3+2 L)\left(\frac{1}{2}\right)^{L}\right) N \\
& N_{\hat{x}}^{\mathrm{mul}}=\sum_{l=1}^{L}\left(\frac{N}{2^{l}}\right)=\left(1-\left(\frac{1}{2}\right)^{L}\right) N .
\end{aligned}
$$

Then, consider the computational complexity of steps 7-9. It can be seen that fixed numbers of additions and multiplications are required in calculating (4), (32) and (22). Therefore, the evaluation of the numbers are straightforward based on these equations. The results are shown in the corresponding columns of Table II.

\section{REFERENCES}

[1] A. Tsiatmas, C. Baggen, F. Willems, J. -P. Linnartz, and J. Bergmans, "An illumination perspective on visible light communications," IEEE Commun. Mag., vol. 52, pp. 64-71, July 2014.

[2] F. Zafar, D. Karunatilaka, and R. Parthiban, "Dimming schemes for visible light communication: the state of research," IEEE Wireless Commun., vol. 22, pp. 29-35, Apr. 2015.

[3] K. Lee and H. Park, "Modulations for Visible Light Communications With Dimming Control," IEEE Photon. Technol. Lett., vol. 23, pp. 11361138, Aug. 2011.

[4] S. Rajagopal, R. D. Roberts, and S. -K. Lim, "IEEE 802.15.7 Visible Light Communication: Modulation Schemes and Dimming Support," IEEE Commun. Mag., vol. 50, pp. 72-82, Mar. 2012.

[5] J. Armstrong et al., "Performance of asymmetrically clipped optical OFDM in AWGN for an intensity modulated direct detection system," in IEEE Globecom, San Francisco, 2006, pp. 1-5.

[6] J. Armstrong and A. J. Lowery, "Power efficient optical OFDM," Electron. Lett., vol. 42, pp. 370-372, Mar. 2006.

[7] J. B. Carruthers and J. M. Kahn, "Multiple-subcarrier modulation for nondirected wireless infrared communication," IEEE J. Sel. Areas Commun., vol. 14, pp. 538-546, Apr. 1996.

[8] S. D. Dissanayake and J. Armstrong, "Comparison of ACO-OFDM, DCO-OFDM and ADO-OFDM in IM/DD Systems," IEEE/OSA J. Lightw. Technol., vol. 31, pp. 1063-1072, Apr. 2013.

[9] D. Tsonev, S. Sinanovic, and H. Haas, "Novel Unipolar Orthogonal Frequency Division Multiplexing (U-OFDM) for Optical Wireless," in IEEE Vehicular Technology Conference (VTC Spring), Yokohama, 2012, pp. 1-5.

[10] S. Dimitrov and H. Haas, "Optimum Signal Shaping in OFDM-based Optical Wireless Communication Systems," in IEEE Vehicular Technology Conference (VTC Fall), Quebec City, 2012, pp. 1-5.

[11] Q. Wang, Z. Wang, and L. Dai, "Asymmetrical hybrid optical OFDM for visible light communications with dimming control," IEEE Photon. Technol. Lett., vol. 27, pp. 974-977, May 2015.

[12] Y. Yang, Z. Zeng, J. Cheng, and C. Guo, "An Enhanced DCO-OFDM Scheme for Dimming Control in Visible Light Communication Systems," IEEE Photonics Journal, vol. 8, June 2016.

[13] G. Ntogari et al., "Combining Illumination Dimming Based on PulseWidth Modulation With Visible-Light Communications Based on Discrete Multitone," IEEE/OSA J. Opt. Commun. Netw., vol. 3, pp. 56-65, Jan. 2011.

[14] H. Elgala and T. D. C. Little, "Reverse polarity optical-OFDM (RPOOFDM): dimming compatible OFDM for gigabit VLC links," Optics Express, vol. 21, pp. 24288-24299, Oct. 2013.

[15] J. -Y. Sung, C. -W. Chow, C. -H. Yeh, and G. -K. Chang, "Twolevel modulation scheme to reduce latency for optical mobile fronthaul networks," Optics Express, vol. 24, pp. 25767-25773, Oct. 2016.

[16] T. Q. Wang and X. Huang, "Dimming Compatible Optical OFDM for High Speed Visible Light Communications," accepted by VTC spring 2017.

[17] Q. Wang, C. Qian, X. Guo, Z. Wang, D. G. Cunningham, and I. H. White, "Layered ACO-OFDM for intensity-modulated directdetection optical wireless transmission," Optics Express, vol. 23, pp. 12382-12393, May 2015. 
[18] B. Ranjha and M. Kavehrad, "Hybrid Asymmetrically Clipped OFDMBased IM/DD Optical Wireless System," IEEE/OSA J. Opt. Commun. Netw., vol. 6, pp. 387-396, Apr. 2014.

[19] D. Tsonev, S. Videv, and H. Haas, "Unlocking Spectral Efficiency in Intensity Modulation and Direct Detection Systems," IEEE J. Sel. Areas Commun., vol. 33, pp. 1758-1770, Sep. 2015.

[20] L. Chen, B. Krongold, and J. Evans, "Successive Decoding of AntiPeriodic OFDM Signals in IM/DD Optical Channel," in Int. Conf. on Communications, Cape Town, 2010, pp. 1-6.

[21] L. Chen, B. Krongold, and J. Evans, "Performance Analysis for Optical OFDM Transmission in Short-Range IM/DD Systems," IEEE/OSA J. Lightw. Technol., vol. 30, pp. 974-983, Apr. 2012.

[22] A. V. Oppenheim et al., Discrete-Time Signal Processing, Upper Saddle River: Prentice-Hall, Inc., 1999.

[23] D. Tsonev, S. Sinanovic, and H. Haas, "Complete Modeling of Nonlinear Distortion in OFDM-Based Optical Wireless Communication," IEEE/OSA J. Lightw. Technol., vol. 31, pp. 3064-3076, Sep. 2013.

[24] M. Tahar, T. Q. Wang, M. F. G. Medina, O. Gonzalez, and J. Armstrong, "Experimental Demonstration of Diversity Combining for Asymmetrically Clipped Optical OFDM,' IEEE Commun. Lett., vol. 20, pp. 906-909, May 2016.

[25] M. Chiani, D. Dardari and M. K. Simon, "New Exponential Bounds and Approximations for the Computation of Error Probability in Fading Channels," IEEE Trans. Wireless Commun., vol. 2, pp. 840-845, July 2003.

[26] S. Hranilovic, Wireless Optical Communication Systems. New York: Springer, 2004.

[27] S. Dimitrov, S. Sinanovic, and H. Haas, "Clipping Noise in OFDMBased Optical Wireless Communication Systems," IEEE Trans. Commun., vol. 60, pp. 1072-1081, Apr. 2012.

[28] A. Papoulis, Probability, random variables, and stochastic processes, New York: Mc-Graw-Hill, 1991.

[29] J. Proakis, Digital Communications, 4th Edition. New York: McGrawHill, 2000.

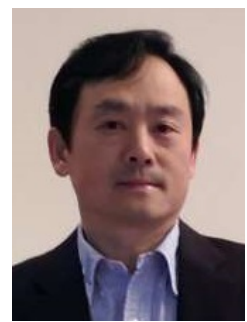

Xiaojing Huang (M 99-SM 11) received the B.E., M.E., and Ph.D. degrees from Shanghai Jiao Tong University, Shanghai, China, in 1983, 1986, and 1989 , respectively, all in electronic engineering. $\mathrm{He}$ is currently a Professor of Information and Communications Technology with the School of Electrical and Data Engineering and the Program Leader for mobile sensing and communications with the Global Big Data Technologies Centre, University of Technology Sydney (UTS), Australia. He had been a Principal Research Scientist with Commonwealth Scientific and Industrial Research Organisation (CSIRO), Australia, and the Project Leader of CSIRO microwave and mm-wave backhaul projects since 2009, an Associate Professor at University of Wollongong, Australia, since 2004, and a Principal Research Engineer with Motorola Australian Research Centre since 1998. With over 29 years of combined industrial, academic, and scientific research experience, he has published over 270 book chapters, refereed journal and conference papers, and major commercial research reports. He has also filed 31 patents. Prof. Huang was a recipient of the CSIRO Chairmans Medal and the Australian Engineering Innovation Award in 2012 for exceptional research achievements in multigigabit wireless communications.

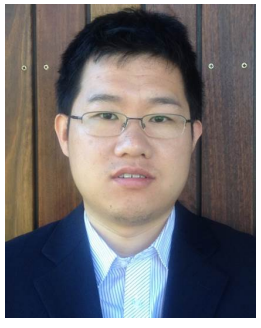

Thomas Q. Wang received B.E. degree in electric engineering from Dalian Jiaotong University, Dalian, China, in 2006, and the M.E. and Ph.D degrees in communication and information systems from Dalian Maritime University, Dalian, China, in 2008 and 2011, respectively. From March 2012 to December 2015, he was a Research Fellow with the Department of Electrical and Computer Systems Engineering, Monash University, Melbourne, VIC, Australia. He is currently a Research Fellow with the Global Big Data Technologies Centre, University of Technology Sydney, Sydney, NSW, Australia. His research interests include optical wireless communications and multiple-input multiple-output technology. 